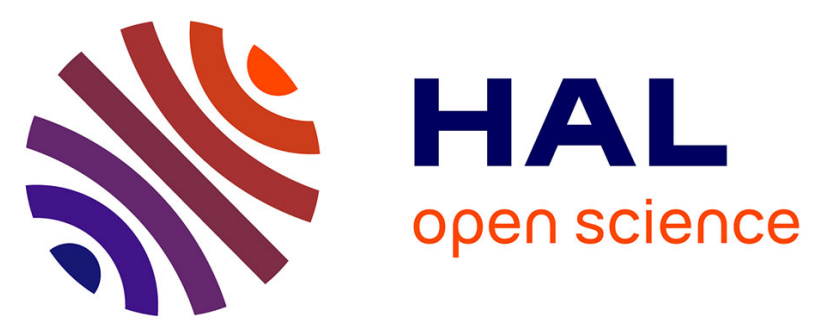

\title{
Isolation and Structural Elucidation of 15-Nuclear Copper Dihydride Clusters: An Intermediate in the Formation of a Two-Electron Copper Superatom
}

Kiran Kumarvarma Chakrahari, Jingping Liao, Rhone P Brocha Silalahi, Tzu-Hao Chiu, Jian-Hong Liao, Xiaoping Wang, Samia Kahlal, Jean-Yves Saillard, C W Liu

\section{To cite this version:}

Kiran Kumarvarma Chakrahari, Jingping Liao, Rhone P Brocha Silalahi, Tzu-Hao Chiu, Jian-Hong Liao, et al.. Isolation and Structural Elucidation of 15-Nuclear Copper Dihydride Clusters: An Intermediate in the Formation of a Two-Electron Copper Superatom. Small, 2021, 17 (27), pp.2002544. 10.1002/smll.202002544 . hal-02998969

\section{HAL Id: hal-02998969 https://hal.science/hal-02998969}

Submitted on 20 Nov 2020

HAL is a multi-disciplinary open access archive for the deposit and dissemination of scientific research documents, whether they are published or not. The documents may come from teaching and research institutions in France or abroad, or from public or private research centers.
L'archive ouverte pluridisciplinaire HAL, est destinée au dépôt et à la diffusion de documents scientifiques de niveau recherche, publiés ou non, émanant des établissements d'enseignement et de recherche français ou étrangers, des laboratoires publics ou privés. 
Isolation and Structural Elucidation of 15-nuclear Copper Dihydride Clusters: An Intermediate in the Formation of a 2-electron Copper Superatom

Kiran Kumarvarma Chakrahari, Jingping Liao, Rhone P. Brocha Silalahi, Tzu-Hao Chiu, Jian-Hong Liao, Xiaoping Wang, * Samia Kahlal, Jean-Yves Saillard,* and C. W. Liu*

Dr. K. K. Chakrahari, ${ }^{\dagger}$ J. Liao, $^{\dagger}$ R. P. B. Silalahi, T.-H. Chiu, Dr. J.-H. Liao, Prof. C. W. Liu

Department of Chemistry, National Dong Hwa University, No. 1, Sec. 2, Da Hsueh Rd. Shoufeng, Hualien 974301, Taiwan (R.O.C.)

E-mail: chenwei@mail.ndhu.edu.tw

Dr. K. K. Chakrahari,

Department of Chemistry, SRM Institute of Science and Technology, Kattankulathur 603203, India

Dr. X. Wang

Neutron Scattering Division, Neutron Sciences Directorate, Oak Ridge National

Laboratory, Oak Ridge, TN 37831, USA

Dr. S. Kahlal, Prof. J.-Y. Saillard

Univ Rennes, CNRS, ISCR-UMR 6226, F-35000 Rennes, France

† The two authors contribute qually to this work.

Keywords: copper, hydride, nanocluster, neutron diffraction

Highly reactive copper-dihydride clusters, $\left[\mathrm{Cu}_{15}(\mathrm{H})_{2}\left(\mathrm{~S}_{2} \mathrm{CNR}_{2}\right)_{6}\left(\mathrm{C}_{2} \mathrm{Ph}\right)_{6}\right]\left(\mathrm{PF}_{6}\right)\{\mathrm{R}=$

$\left.{ }^{n} \mathrm{Bu}\left(\mathbf{1}_{\mathbf{H}}\right),{ }^{n} \operatorname{Pr}\left(\mathbf{2}_{\mathbf{H}}\right),{ }^{i} \mathrm{Bu}\left(\mathbf{3}_{\mathbf{H}}\right)\right\}$, were isolated during the reaction of

$\left[\mathrm{Cu}_{28} \mathrm{H}_{15}\left\{\mathrm{~S}_{2} \mathrm{CN}^{n} \mathrm{Bu}_{2}\right\}_{12}\right]\left(\mathrm{PF}_{6}\right)$ with ten equivalents of phenylacetylene. They are found to be intermediates in the formation of the earlier reported 2-electron superatom $\left[\mathrm{Cu}_{13}\left(\mathrm{~S}_{2} \mathrm{CNR}_{2}\right)_{6}\left(\mathrm{C}_{2} \mathrm{Ph}\right)_{4}\right]^{+}$. Better yields were obtained by reacting dithiocarbamate sodium salts, $\left[\mathrm{Cu}\left(\mathrm{CH}_{3} \mathrm{CN}\right)_{4}\right]\left(\mathrm{PF}_{6}\right), \mathrm{BH}_{4}{ }^{-}$and phenylacetylene. The presence of two hydrides in $\mathbf{1}_{\mathbf{H}}$ was confirmed by the synthesis and characterization of its deuteride analogue $\left[\mathrm{Cu}_{15}(\mathrm{D})_{2}\left(\mathrm{~S}_{2} \mathrm{CN}^{n} \mathrm{Bu}_{2}\right)_{6}\left(\mathrm{C}_{2} \mathrm{Ph}\right)_{6}\right]^{+}, \mathbf{1}_{\mathbf{D}}$, and a single crystal neutron structure of $\mathbf{2}_{\mathbf{H}}$. Structural characterization of $\mathbf{1}_{\mathbf{H}}$ revealed a new bicapped icosahedral copper(I) 
cage encapsulating a linear copper dihydride $\left(\mathrm{CuH}_{2}\right)^{-}$unit. Reaction of $\mathbf{3}_{\mathbf{H}}$ with $\mathrm{Au}(\mathrm{I})$ salts yielded a highly luminescent $\left[\mathrm{AuCu}_{12}\left(\mathrm{~S}_{2} \mathrm{CN}^{i} \mathrm{Bu}_{2}\right)_{6}\left(\mathrm{C}_{2} \mathrm{Ph}\right)_{4}\right]^{+}$cluster.

The chemistry of transition-metal hydride clusters has recently received significant interest. $^{[1,2]}$ Such compounds play a key role as intermediates in several homogeneous and heterogeneous catalyses ${ }^{[3]}$ with significant contributions in hydrogen storage. ${ }^{[4]}$ Importantly, copper hydride chemistry has evolved towards the design of nanosized ligand-protected polyhydrido copper clusters that have emerged as a new class of materials in the field of nanoscience, owing to their various potential applications. ${ }^{\text {[2f-h] }}$ Our group has made significant contributions in this endeavor by isolated a series of air-stable copper(I) monohydride clusters stabilized by 1,1-dichalcogenolates. ${ }^{[5]}$ Subsequently four nanoscale copper polyhydrides, $\left[\mathrm{Cu}_{20} \mathrm{H}_{11}\left\{\mathrm{E}_{2} \mathrm{P}\left(\mathrm{O}^{i} \mathrm{Pr}\right)_{2}\right\}_{9}\right](\mathrm{E}=\mathrm{S}$, $\mathrm{Se}),{ }^{[6]}\left[\mathrm{Cu}_{28}(\mathrm{H})_{15}\left(\mathrm{~S}_{2} \mathrm{CNR}_{2}\right)_{12}\right] \mathrm{PF}_{6},{ }^{[7]}\left[\mathrm{Cu}_{30} \mathrm{H}_{18}\left\{\mathrm{~S}_{2} \mathrm{P}\left(\mathrm{O}^{\mathrm{n}} \mathrm{Pr}\right)_{2}\right\}_{12}\right]^{[8]}$ and $\left[\mathrm{Cu}_{32}(\mathrm{H})_{20}\left\{\mathrm{~S}_{2} \mathrm{P}\left(\mathrm{O}^{i} \mathrm{Pr}\right)_{2}\right\}_{12}\right]^{[9]}$ were structurally characterized by both X-ray and neutron diffraction from which a new synthetic concept has been established, based on the fact that hydride additions assist the growth of nanoscale copper clusters. Indeed, these hydrides have been found to be intermediates in the fabrication of rhombus-shaped copper nanoparticles via a reduction route as well as electrocatalysts in the $\mathrm{CO}_{2}$ reduction. ${ }^{[9 b]}$

In recent years, alkynyls have emerged as promising ligands for stabilizing metal nanoclusters. ${ }^{[10-14]}$ Alkynyl-protected Au-Ag alloy nanoclusters (NCs) have been isolated. ${ }^{[11-14]}$ Synthesis of copper NCs by reacting $\left[\mathrm{Cu}_{28}(\mathrm{H})_{15}(\mathrm{dtc})_{12}\right]^{+}(\mathrm{dtc}=\mathrm{di}-$ butyldithiocarbamate, $\mathrm{S}_{2} \mathrm{CN}^{n} \mathrm{Bu}_{2}$ ) with terminal alkynes was established, where the hydrides present in the $\mathrm{Cu}(\mathrm{I})$ cluster react with the acidic protons of the terminal alkynes, liberating $\mathrm{H}_{2}$ under elevated temperature, which subsequently reduces the 
leftover of alkynes to alkenes and some of $\mathrm{Cu}(\mathrm{I})$ to $\mathrm{Cu}(0) .{ }^{[15]}$ The participation of alkynyls in the reaction medium leads eventually to the formation of the brand-new $\mathrm{NC}\left[\mathrm{Cu}_{13}(\mathrm{dtc})_{6}(\text { alkynyl })_{4}\right]^{+}$, a 2-electron superatom. To explore this chemistry further, it is imperative to understand the mechanism involved in the formation of these superatomic clusters. A similar reaction in the presence of $\mathrm{Pd}\left(\mathrm{PPh}_{3}\right)_{2} \mathrm{Cl}_{2}$ yielded $\left[\mathrm{PdH}_{2} @ \mathrm{Cu}_{14}\left\{\mathrm{~S}_{2} \mathrm{CN}^{\mathrm{n}} \mathrm{Bu}_{2}\right\}_{6}\{\mathrm{C} \equiv \mathrm{CPh}\}_{6}\right] .^{[16]}$ This serendipitous result gave us a hint of a potentially isolable intermediate $\left[\mathrm{CuH}_{2} @ \mathrm{Cu}_{14}\left\{\mathrm{~S}_{2} \mathrm{CN}^{n} \mathrm{Bu}_{2}\right\}_{6}\{\mathrm{C} \equiv \mathrm{CPh}\}_{6}\right]^{+}$during the formation of $\left[\mathrm{Cu}_{13}(\mathrm{dtc})_{6}(\mathrm{alkynyl})_{4}\right]^{+}$. Here, we have successfully isolated and structurally characterized both hydride and deuteride analogs of such clusters, which not only depict a linear $\left[\mathrm{CuH}_{2}\right]^{-}$complex embeded in a $\left[\mathrm{Cu}_{14}\right]^{14+}$ cage, but also readily convert to $\left[\mathrm{Cu}_{13}\right]^{11+}$ at elevated temperatures.

In a typical experiment, to a THF suspension of $\left[\mathrm{Cu}_{28} \mathrm{H}_{15}\left(\mathrm{~S}_{2} \mathrm{CN}^{\mathrm{n}} \mathrm{Bu}_{2}\right)_{12}\right]^{+}$, a ten-fold excess of phenylacetylene was added and the reaction mixture was stirred at $30{ }^{\circ} \mathrm{C}$ for 12 hours. The color of the suspension changed from orange to red. Workup of this mixture leads to the isolation of $\left[\mathrm{Cu}_{15}(\mathrm{H})_{2}\left\{\mathrm{~S}_{2} \mathrm{CN}^{n} \mathrm{Bu}_{2}\right\}_{6}\{\mathrm{C} \equiv \mathrm{CPh}\}_{6}\right]^{+}\left(\mathbf{1}_{\mathbf{H}}\right)$ as an orange-yellow solid in $20 \%$ yield, along with $\left[\mathrm{Cu}_{8} \mathrm{H}\left(\mathrm{S}_{2} \mathrm{CN}^{n} \mathrm{Bu}_{2}\right)_{6}\right]^{+}$and styrene as byproducts. The yield of $\mathbf{1}_{\mathbf{H}}$ formation could be increased (70\%) by an alternating strategy, from the mixture of $\mathrm{Cu}(\mathrm{I})$, dithiocarbamate, $\mathrm{NaBH}_{4}$, phenylacetylene and $\mathrm{NEt}_{3}$ in the 5:2:1:8:8 ratio, respectively (Scheme 1). The corresponding deuteride derivative $\left[\mathrm{Cu}_{15}(\mathrm{D})_{2}\left(\mathrm{~S}_{2} \mathrm{CN}^{n} \mathrm{Bu}_{2}\right)_{6}\left(\mathrm{C}_{2} \mathrm{Ph}\right)_{6}\right]\left(\mathrm{PF}_{6}\right)\left(\mathbf{1}_{\mathbf{D}}\right)(73 \%)$ was synthesized to support the presence of the hydride ligands in $\mathbf{1}_{\mathbf{H}}$ through ${ }^{1} \mathrm{H}$ NMR studies. By using different carbamates, we have isolated analogous hydride and deuteride derivatives of $\mathbf{1}_{\mathbf{H}}$; namely $\left[\mathrm{Cu}_{15}(\mathrm{H} / \mathrm{D})_{2}\left(\mathrm{~S}_{2} \mathrm{CN}^{n} \mathrm{Pr}_{2}\right)_{6}\left(\mathrm{C}_{2} \mathrm{Ph}\right)_{6}\right]\left(\mathrm{PF}_{6}\right)\left(\mathbf{2}_{\mathbf{H}^{-}} \mathbf{6 5 \%} / \mathbf{2}_{\mathbf{D}}-65 \%\right)$ and $\left[\mathrm{Cu}_{15}(\mathrm{H} / \mathrm{D})_{2}\left(\mathrm{~S}_{2} \mathrm{CN}^{i} \mathrm{Bu}_{2}\right)_{6}\left(\mathrm{C}_{2} \mathrm{Ph}\right)_{6}\right]\left(\mathrm{PF}_{6}\right),\left(\mathbf{3}_{\mathbf{H}^{-}} \mathbf{7 4 \%} / \mathbf{3}_{\mathbf{D}^{-}} \mathbf{7 5 \%}\right)$. Their full characterization 
was established by ESI-MS spectrometry, NMR spectroscopy, single crystal X-ray diffraction analysis and neutron diffraction (in the case of $\mathbf{2}_{\mathbf{H}}$ ).

The positive-ion ESI spectrum of $\mathbf{1}_{\mathbf{H}}$ displays three characteristic peaks corresponding to $\left[\mathbf{1}_{\mathbf{H}}\right]^{+}$at $\mathrm{m} / \mathrm{z} 2786.72$ (calc. 2787.72 ), its fragment ions $\left[\mathbf{1}_{\mathbf{H}^{-}}-\mathrm{Cu}\left(\mathrm{C}_{2} \mathrm{Ph}\right)\right]^{+}$at 2620.74 (calc. 2621.74) and $\left[\mathbf{1}_{\mathbf{H}^{-}}\left\{\mathrm{CuC}_{2} \mathrm{Ph}\right\}_{2}\right]^{+}$at 2456.77 (calc. 2456.77) (Figure 1). The latter two species correspond to a successive loss of one and two $\left[\mathrm{Cu}\left(\mathrm{C}_{2} \mathrm{Ph}\right)\right]$ fragments from $\mathbf{1}_{\mathbf{H}}$ in the gas phase. The ESI-MS analysis of the deuteride analogue $\left[\mathrm{Cu}_{15}(\mathrm{D})_{2}\left\{\mathrm{~S}_{2} \mathrm{CN}^{n} \mathrm{Bu}_{2}\right\}_{6}\left(\mathrm{C}_{2} \mathrm{Ph}\right)_{6}\right]\left(\mathrm{PF}_{6}\right)\left(\mathbf{1}_{\mathbf{D}}\right)$ confirmed the presence of two deuterides by showing a band at $\mathrm{m} / \mathrm{z} 2788.72$ (calc.2789.72) (Figure S1). Their theoretically determined isotopic patterns show good resemblance with the experimental ones, as shown in Figure S1 (inset). The deviation between the $\left[\mathbf{1}_{\mathbf{H}}\right]^{+}$and $\left[\mathbf{1}_{\mathbf{D}}\right]^{+}$peaks indicates the existence of 2 hydrides in $\left[\mathbf{1}_{\mathbf{H}}\right]^{+}$. The positve-ion ESI-MS spectra of $\mathbf{2}_{\mathbf{H}}$ (Figure S2) and $\mathbf{3}_{\mathbf{H}}$ (Figure S3) also show identical bands for $\left[\mathbf{2}_{\mathbf{H}}\right]^{+}$and $\left[\mathbf{3}_{\mathbf{H}}\right]^{+}$, at $\mathrm{m} / \mathrm{z} 2618.5$ (calc. 2116.5), and 2786.7 (calc. 2787.7), respectively, along with fragment ions by losing $\left\{\mathrm{Cu}\left(\mathrm{C}_{2} \mathrm{Ph}\right)\right\}$ and $\left\{\mathrm{Cu}\left(\mathrm{C}_{2} \mathrm{Ph}\right)\right\}_{2}$ moieties. The ESI-MS results of $\mathbf{2}_{\mathbf{D}}$ and $\mathbf{3}_{\mathbf{D}}$ are in good agreement with the calculated patterns (Figure S4, S5).

The ${ }^{1} \mathrm{H}$ NMR spectrum of $\mathbf{1}_{\mathbf{H}}$ (Figure S6a) shows the presence of two hydrides, six dithiocarbamates and six phenyl ligands. The two hydrides are associated with a resonance peak at $5.07 \mathrm{ppm}$ in $\mathrm{CD}_{2} \mathrm{Cl}_{2}$, which is echoed by the presence of deuteride signal at $4.98 \mathrm{ppm}$ for compound $\mathbf{1}_{\mathbf{D}}$ in $\mathrm{CH}_{2} \mathrm{Cl}_{2}$ (Figure S6b). Similarly, the hydride and deuteride resonance for $\mathbf{2}_{\mathbf{H}} / \mathbf{2}_{\mathbf{D}}$ and $\mathbf{3}_{\mathbf{H}} / \mathbf{3}_{\mathbf{D}}$ appear at 4.93/4.86 and 4.99/5.13 ppm respectively, in $\mathrm{CDCl}_{3} / \mathrm{CHCl}_{3}$ (Figure S7-S10).

Single crystals of $\mathbf{1}_{\mathbf{H}}, \mathbf{2}_{\mathbf{H}}$ and $\mathbf{3}_{\mathbf{H}}$ were grown by vapour diffusion of hexanes into a concentrated acetone solution at $5{ }^{\circ} \mathrm{C}$. The $\mathrm{X}$-ray structures of $\mathbf{1}_{\mathbf{H}}-\mathbf{3}_{\mathbf{H}}$ and the neutron structure of $\mathbf{2}_{\mathbf{H}}$ are similar. They exhibit a linear $\mathrm{CuH}_{2}$ unit (Figure 2, S11a) 
encapsulated within a $\mathrm{Cu}_{14}$ bicapped icosahedron (Table S1 and S2). The two capping coppers $\left(\mathrm{Cu}_{\text {cap }}\right)$ and the $\mathrm{CuH}_{2}\left(\mathrm{Cu}_{\text {cent }} \mathrm{H}_{2}\right)$ unit are aligned along a $C_{3}$ axis so that the $\mathrm{Cu}_{15} \mathrm{H}_{2}$ core adopts $D_{3 d}$ ideal symmetry (Figure $\mathrm{S} 12$ ). Metrical data are given in Table S3. The hydride positions are fully ascertained by the neutron diffraction structure of $\mathbf{2}_{\mathbf{H}}$, which exhibits a short $\mathrm{Cu}_{\text {cent }}-\mathrm{H}$ average distance (1.78(2) $\AA$ ). Each hydride is located within a trigonal-bypiramidal cavity formed by three icosahedral $\mathrm{Cu}$ atoms

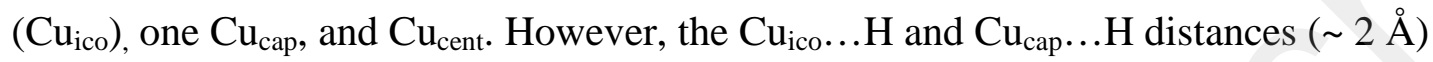
are much longer than their $\mathrm{Cu}_{\text {cent }}-\mathrm{H}$ counterparts (Table S3). The $\left[\left(\mathrm{CuH}_{2}\right) @ \mathrm{Cu}_{14}\right]$ core is stabilized by six dithiocarbamates and six phenylacetylenes (Figure S11d). The dithiocarbamates are equally distributed on the cluster top and bottom, along the $\mathrm{C}_{3}$ axis. They bridge four metal centers (one $\mathrm{Cu}_{\text {cap }}$ and three $\left.\mathrm{Cu}_{\mathrm{ico}}\right)$ in a $\left(\mu_{2}, \mu_{2}\right)$ binding mode. The phenylacetylenes are arranged along the waist of the copper icosahedron, alternatively binding at the top and bottom $\mathrm{Cu}_{\text {ico }}$ triangles in $\mu_{3}$ fashion. As a result, the ideal $D_{3 d}$ symmetry of the $\mathrm{Cu}_{15} \mathrm{H}_{2}$ core is reduced to $S_{6}$.

The UV-Vis spectrum of the $\left[\mathrm{Cu}_{15}(\mathrm{H})_{2}\left\{\mathrm{~S}_{2} \mathrm{CNR}_{2}\right\}_{6}\left(\mathrm{C}_{2} \mathrm{Ph}\right)_{6}\right]^{+}$NCs show broad multiband absorption peaks at 509, 540, and $570 \mathrm{~nm}$ and an intense absorption band at $310 \mathrm{~nm}$. The stability of $\mathbf{2}_{\mathbf{H}}$ in chloroform at ambient temperature was studied by UVVis spectra. The absorption spectrum changed steadily over time and within 24 hours a new band appeared at $360 \mathrm{~nm}$, corresponding to $\left[\mathrm{Cu}_{13}\left\{\mathrm{~S}_{2} \mathrm{CN}^{n} \mathrm{Pr}_{2}\right\}_{6}\left(\mathrm{C}_{2} \mathrm{Ph}\right)_{4}\right]^{+}$(Figure S13). ${ }^{[15]}$ Reaction of $\mathbf{3}_{\mathbf{H}}$ with $\mathrm{Au}\left(\mathrm{PPh}_{3}\right) \mathrm{Cl}$ in dichloromethane for one hour at room temperature yielded a highly luminescent $\left[\mathrm{AuCu}_{12}\left(\mathrm{~S}_{2} \mathrm{CN}^{i} \mathrm{Bu}_{2}\right)_{6}\left(\mathrm{C}_{2} \mathrm{Ph}\right)_{4}\right]^{+}$cluster

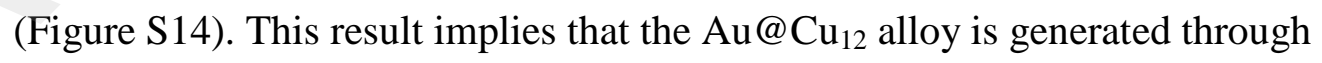
formation of $\left[\mathrm{Cu}_{13}\left\{\mathrm{~S}_{2} \mathrm{CN}^{i} \mathrm{Bu}_{2}\right\}_{6}\left(\mathrm{C}_{2} \mathrm{Ph}\right)_{4}\right]^{+}$. 17$]^{-17}$

DFT calculations were performed on a simplified model of 1-3 (see SI), namely $\left[\mathrm{Cu}_{15}(\mathrm{H})_{2}\left(\mathrm{~S}_{2} \mathrm{CNH}_{2}\right)_{6}\left(\mathrm{C}_{2} \mathrm{Ph}\right)_{6}\right]^{+}\left(\mathbf{1}^{\prime}\right)$. Its optimized geometry is of $C_{i}$ symmetry, slightly 
distorted away from $S_{6}$. Its metric data are in good agreement with the X-ray structures of 1-3 (Table S3). It has a large HOMO-LUMO gap (3.90 eV), as expected for a stable $\mathrm{Cu}(\mathrm{I})$ species. Consistently, the copper natural atomic orbital (NAO) charges are substantially positive $\left(0.29,0.77\right.$ (av.) and 0.66 for $\mathrm{Cu}_{\text {cent }}, \mathrm{Cu}_{\text {ico }}$ and $\mathrm{Cu}_{\text {cap }}$, respectively. On the other hand, the hydride NAO charges are negative $(-0.62)$. The small values of the $\mathrm{Cu}-\mathrm{Cu}$ Wiberg indices ( $<0.07$, see Table S3) agree with the view of weakly interacting $\mathrm{Cu}(\mathrm{I})$ centers. The $\mathrm{Cu}_{\text {cap }}-\mathrm{H}$ and $\mathrm{Cu}_{\text {ico }}-\mathrm{H}$ Wiberg indices (av. 0.055) are much smaller than their $\mathrm{Cu}_{\text {cent }}-\mathrm{H}$ counterpart (0.164), in line with the corresponding interatomic distances. These data are consistent with the view of a linear 14-electron $\left[\mathrm{CuH}_{2}\right]^{-}$complex encapsulated within a $\left[\mathrm{Cu}_{14}\right]^{14+}$ cage. ${ }^{[18]}$ Similar calculations on the free $\left[\mathrm{CuH}_{2}\right]^{-}$anion led to $\mathrm{Cu}-\mathrm{H}$ distances, $\mathrm{Cu}-\mathrm{H}$ Wiberg indices and Cu NAO charge of $1.571 \AA, 0.486$, and 0.21 , respectively. These values support the view of a strong ionic interaction between $\left[\mathrm{CuH}_{2}\right]^{-}$and its cage. TD-DFT calculations on 1' (Figure S15) were unable to reproduce the low energy band. The intense band was found originating from three major transitions computed at 385, 364 and $306 \mathrm{~nm}$ and corresponding to $\mathrm{dtc} \rightarrow \mathrm{Cu}_{15}$ charge transfer.

The degradation of $\mathbf{2}_{\mathbf{H}}$ was investigated by variable temperature ${ }^{1} \mathrm{H}$ NMR (25 to 50 ${ }^{\circ} \mathrm{C}$ ) in $\mathrm{CDCl}_{3}$ (Figure S16). The results show good agreement with our UV-vis studies, where $2_{\mathbf{H}}$ was converted to $\left[\mathrm{Cu}_{13}\left\{\mathrm{~S}_{2} \mathrm{CN}^{n} \mathrm{Pr}_{2}\right\}_{6}\left(\mathrm{C}_{2} \mathrm{Ph}\right)_{4}\right]^{+}$by the liberation of $\mathrm{H}_{2}$ (peak at 4.8 ppm). This indicates that $\left[\mathrm{Cu}_{15}(\mathrm{H})_{2}\left\{\mathrm{~S}_{2} \mathrm{CN}^{n} \mathrm{Pr}_{2}\right\}_{6}\left(\mathrm{C}_{2} \mathrm{Ph}\right)_{6}\right]^{+}$is unstable in chlorinated solvents at high temperature. This is supported by ESI-MS recorded by using dichloromethane as solvent (Figure S17). We suggest (Scheme 2) that $\mathbf{2}_{\mathbf{H}}(\mathrm{m} / \mathbf{z}=$ 2618.8) disintegrates to $\left[\mathrm{Cu}_{14}(\mathrm{H})_{2}\left\{\mathrm{~S}_{2} \mathrm{CN}^{n} \mathrm{Pr}_{2}\right\}_{6}\left(\mathrm{C}_{2} \mathrm{Ph}\right)_{5}\right]^{+}(\mathrm{m} / \mathrm{z}=2452.5)$ by the loss of $\left[\mathrm{Cu}\left(\mathrm{C}_{2} \mathrm{Ph}\right)\right]$. The disintegration continues to peel away another $\left[\mathrm{Cu}\left(\mathrm{C}_{2} \mathrm{Ph}\right)\right]$, leading to $\left[\mathrm{Cu}_{13} \mathrm{H}_{2}\left\{\mathrm{~S}_{2} \mathrm{CN}^{n} \mathrm{Pr}_{2}\right\}_{6}\left(\mathrm{C}_{2} \mathrm{Ph}\right)_{4}\right]^{+}$and finally releasing $\mathrm{H}_{2}$ to form the 2-electron 
superatom $\left[\mathrm{Cu}_{13}\left\{\mathrm{~S}_{2} \mathrm{CN}^{n} \mathrm{Pr}_{2}\right\}_{6}\left(\mathrm{C}_{2} \mathrm{Ph}\right)_{4}\right]^{+}(\mathrm{m} / \mathrm{z}=2288.5)$. The last stage of decomposition is possible via an internal redox reaction;

$\left(\mathrm{C}^{\mathrm{u}^{+}}+2 \mathrm{H}^{-} \longrightarrow \mathrm{C}^{-1}+\mathrm{H}_{2}\right)$, which is accompanied with icosahedron to cuboctahedron structural rearrangement of the centered $\mathrm{Cu}_{13}$ unit (Figure S18). This is supported by DFT calculations that found stable structures of $\left[\mathrm{Cu}_{14}(\mathrm{H})_{2}\left(\mathrm{~S}_{2} \mathrm{CNR}_{2}\right)_{6}\left(\mathrm{C}_{2} \mathrm{Ph}\right)_{5}\right]^{+}$and $\left[\mathrm{Cu}_{13}(\mathrm{H})_{2}\left(\mathrm{~S}_{2} \mathrm{CNR}_{2}\right)_{5}\left(\mathrm{C}_{2} \mathrm{Ph}\right)_{4}\right]^{+}$compositions as potential intermediates (see SI). We thus propose that $\left[\mathrm{Cu}_{15}(\mathrm{H})_{2}\left\{\mathrm{~S}_{2} \mathrm{CNR}_{2}\right\}_{6}\left(\mathrm{C}_{2} \mathrm{Ph}\right)_{6}\right]^{+}$ is an intermediate product which releases external energy to form the stable $\left[\mathrm{Cu}_{13}\left\{\mathrm{~S}_{2} \mathrm{CNR}_{2}\right\}_{6}\left(\mathrm{C}_{2} \mathrm{Ph}\right)_{4}\right]^{+} \mathrm{NC}$. Here the reducing agents yielding $\mathrm{Cu}^{0}$ from $\mathrm{Cu}^{\mathrm{I}}$ are the hydrides.

In summary, we isolated the highly reactive clusters $\left[\mathrm{Cu}_{15}(\mathrm{H})_{2}\left(\mathrm{~S}_{2} \mathrm{CNR}_{2}\right)_{6}\left(\mathrm{C}_{2} \mathrm{Ph}\right)_{6}\right]\left(\mathrm{PF}_{6}\right), \mathrm{R}={ }^{n} \mathrm{Bu}\left(\mathbf{1}_{\mathbf{H}}\right),{ }^{n} \operatorname{Pr}\left(\mathbf{2}_{\mathbf{H}}\right)$ and ${ }^{i} \mathrm{Bu}\left(\mathbf{3}_{\mathbf{H}}\right)$, which helps understanding the intermediates involved in the formation of a 2-electron copper superatom. The reaction was monitored and the intermediates were fully characterized. Futhermore, in $\mathrm{CDCl}_{3}$ the $\left[\mathrm{Cu}_{15}(\mathrm{H})_{2}\left(\mathrm{~S}_{2} \mathrm{CNR}_{2}\right)_{6}\left(\mathrm{C}_{2} \mathrm{Ph}\right)_{6}\right]\left(\mathrm{PF}_{6}\right)$ undergoes degradation to release two $\left[\mathrm{Cu}\left(\mathrm{C}_{2} \mathrm{Ph}\right)\right]$ to form $\left[\mathrm{Cu}_{13} \mathrm{H}_{2}\left\{\mathrm{~S}_{2} \mathrm{CNR}_{2}\right\}_{6}\left(\mathrm{C}_{2} \mathrm{Ph}\right)_{4}\right]^{+}$, which finally releases $\mathrm{H}_{2}$ via a redox reaction to form superatom clusters.

\section{Supporting Information}

Supporting Information is available from the Wiley Online Library.

\section{Acknowledgements}

This work was supported by the Ministry of Science and Technology of Taiwan (MOST 109 - 2113 - M - 259 - 008, 108 - 2923 - M - 259 - 001), and the France Taiwan ANR - MOST program (project Nanoalloys). KKC would like to thank SERB for financial support (SRG/2019/001618). The GENCI is acknowledged for HPC resources (Project A0050807367). Single crystal neutron diffraction used resources at the Spallation Neutron Source, a DOE Office of Science User Facility operated by the Oak Ridge National Laboratory, under Contract No. DE-AC0500OR22725 with UT-Battelle, LLC. This manuscript has been authored by UTBattelle, LLC, under contract DE-AC05-00OR22725 with the US Department of Energy (DOE). The US government retains and the publisher, by accepting the article for publication, acknowledges that the US government retains a nonexclusive, paid-up, 
irrevocable, worldwide license to publish or reproduce the published form of this manuscript, or allow others to do so, for US government purposes. DOE will provide public access to these results of federally sponsored research in accordance with the DOE Public Access Plan (http://energy.gov/downloads/doe-public-access-plan)

Received: ((will be filled in by the editorial staff))

Revised: ((will be filled in by the editorial staff)) Published online: ((will be filled in by the editorial staff))

\section{References}

R. Poli, M. Peruzzini, In Recent Advances in Hydride Chemistry 1st Edition.

Elsevier: Amsterdam, 2001, p 557.

[2] a) F. Maseras, A. Lledos, E. Clot, O. Eisenstein, Chem. Rev., 2000, 100, 601-

636; b) R. B. King, Coord. Chem. Rev, 2000, 200-202, 813-829; c) A.J. Hoskin, D.

W. Stephan, Coord. Chem. Rev., 2002, 233-234, 107-129; d) R. H. Morris, Coord.

Chem. Rev, 2008, 252, 2381-2394; e) P. L. Holland, Acc. Chem. Res., 2008, 41, 905-

914; f) R. S. Dhayal, W. E. van Zyl, C. W. Liu, Acc. Chem. Res. 2016, 49, 86-95; g)

A. J. Jordan, G. Lalic, J. P. Sadighi, Chem. Rev. 2016, 116, 8318-8372; h) T.

Nakajima, K. Nakamae, Y, Ura, T. Tanase, Eur. J. Inorg. Chem. 2020, 2211-2226.

[3] a) B. Cornils, W. A. Herrmann, In Applied Homogeneous Catalysis with

Organometallic Compounds; Wiley-VCH: Weinheim, Germany, 1996; b) S. Gaillard,

J.-L. Renaud, ChemSusChem., 2008, 1, 505-509.

[4] a) K. Yvon, G. Renaudin, Hydrides: Solid State Transition Metal Complexes.

In Encyclopedia of Inorganic Chemistry, 2nd ed.; King R. B., Ed., Wiley: Chichester, U.K., 2005; Vol. 3, p 1814; b) J. Graetz, Chem. Soc. Rev., 2009, 38, 73-82; c) J. Yang, A. Sudik, C. Wolverton, D. J. Siegel, Chem. Soc. Rev., 2010, 39, 656-675. d) R. S.

Dhayal, W. E. van Zyl, C. W. Liu, Dalton Trans., 2019, 48, 3531 - 3538. 
[5] a) C. W. Liu, B. Sarkar, Y. -J. Huang, P. -K. Liao, J.-C. Wang, J. -Y. Saillard, S. Kahal, J. Am. Chem. Soc., 2009, 131, 11222-11233; b) P. -K. Liao, B. Sarkar, H. W. Chang, J. -C. Wang, C. W. Liu,. Inorg. Chem., 2009, 48, 4089-4097; c) P. -K. Liao, K. -G. Liu, C. -S. Fang, C. W. Liu, J. P. Jr. Fackler, Y. -Y. Wu, Inorg. Chem., 2011, 50, 8410-8417; d) P. -K. Liao, C. -S. Fang, A. J. Edwards, S. Kahlal, J. -Y. Saillard, C. W. Liu, Inorg. Chem., 2012, 51, 6577-6591.

[6] a) R. S. Dhayal, J. -H. Liao, X. Wang, Y. -C. Liu, M. -H. Chiang, S. Kahlal, J. -Y. Saillard, C. W. Liu, Angew. Chem. Int. Ed, 2015, 54, 13604 - 13608; b) R. S. Dhayal, J. -H. Liao, Y. -R. Lin, P. -K. Liao, S. Kahlal, J. -Y. Saillard, C. W. Liu, J. Am. Chem. Soc., 2013, 135, 4704 - 4707; c) J. -H. Liao, R. S. Dhayal, X. Wang, S. Kahlal, J. -Y. Saillard, C. W. Liu, Inorg. Chem., 2014, 53, 11140 - 11145; d) P.-Y. Lin, D.-Y. Li, F.-H. Ho; J.-H. Liao, S. K. Barik, C. W. Liu, J. Chin. Chem. Soc., 2019, 66, 988-995.

[7] a) P. V. V. N. Kishore, J. -H. Liao, H. -N. Hou, Y. -R. Lin, C. W. Liu, Inorg. Chem., 2016, 55, 3663 - 3673; b) A. J. Edwards, R. S. Dhayal, P. -K. Liao, J. -H. Liao, M. -H. Chiang, R. O. Piltz, S. Kahlal, J. -Y. Saillard, C. W. Liu, Angew. Chem. Int. Ed, 2014, 53, $7214-7218$.

[8] S. K. Barik, S. -C. Huo, C. -Y. Wu, T. -Z. Chiu, J. -H. Liao, X. Wang, S. Kahlal, J. -Y. Saillard, C. W. Liu, Chem. Eur. J., doi.org/10.1002/chem.202001449; [9] a) R. S. Dhayal, J. -H. Liao, S. Kahlal, X. Wang, Y. -C. Liu, M. -H. Chiang, W. E. van Zyl, J. -Y. Saillard, C. W. Liu, Chem. Eur. J., 2015, 21, 8369 - 8674; b) Q. Tang, Y. Lee, D.-Y. Li, W. Choi, C. W. Liu, D. Lee, D.-e. Jiang, J. Am. Chem. Soc. 2017, 139, 9728-9736; c) R. S. Dhayal, H. -P. Chen, J. -H. Liao, W. E. Van Zyl, C. W. Liu, ChemistrySelect, 2018, 3, $3603-3610$. 
[10] a) Z. Lei, X.-K. Wan, S.-F. Yuan, J.-Q. Wang, Q. -M. Wang, Q.-M. Dalton Trans., 2017, 46, 3427-3434; b) X. -K. Wan, W. -W. Xu, S. -F. Yuan, Y. Gao, X. Zeng, Q.-M. Wang, Angew. Chem. Int. Ed., 2015, 54, 9683-9686; c) Z.-J. Guan, J.-L. Zeng, Z.-A. Nan, X.-K. Wan, Y.-M. Lin, Q.-M. Wang, Sci. Adv., 2016, 2, e1600323; d) S.-F. Yuan, P. Li, Q. Tang, X.-K. Wan, Z.-A. Nan, D.-e. Jiang, Q.-M. Wang, Nanoscale, 2017, 9, 11405-11409.

[11] a) N. Kobayashi, Y. Kamei, Y. Shichibu, K. Konishi, J. Am. Chem. Soc., 2013, 135, 16078-16081; b) M. Qu, H. Li, L. H. Xie, S. -T. Yan, J. -R. Li, J. -H. Wang, C. -Y. Wei, Y. -W. Wu, X. -M. Zhang, J. Am. Chem. Soc., 2017, 139, 12346-12349. [12] a) Y. Wang, H. Su, C. Xu, G. Li, L. Gell, S. Lin, Z. Tang, H. Häkkinen, N. Zheng, J. Am. Chem. Soc., 2015, 137, 4324-4327; b) Y. Wang, X.-K. Wan, L, Ren, H. Su, G. Li, S. Malola, S. Lin, Z. Tang, H. Häkkinen, B. K. Teo, Q. -M. Wang, N. Zheng, J. Am. Chem. Soc., 2016, 138, 3278-3281.

[13] a) X. -K. Wan, X. -L. Cheng, Q. Tang, Y. -Z. Han, G. Hu, G, D.-e. Jiang, Q. -M. Wang, J. Am. Chem. Soc., 2017, 139, 9451-9454; b) H. Shen, T. Mizuta, Chem. Asian J., 2017, 12, 2904-2907.

[14] Y. Du, Z. -J. Guan, Z. -R. Wen, Y. -M. Lin, Q. -M. Wang, Chem. Eur. J., 2018, 24, 16029-16035.

[15] a) K. K. Chakrahari, J.-H. Liao, S. Kahlal, Y.-C. Liu, M. -H. Chiang, J.-Y. Saillard, C. W. Liu, Angew. Chem. Int. Ed., 2016, 55, 14704 - 14708; b) a) K. K. Chakrahari, R. P. B. Silalahi, J.-H. Liao, S. Kahlal, Y.-C. Liu, J.-F. Lee, M. -H. Chiang, J.-Y. Saillard, C. W. Liu, Chem. Sci., 2018, 9, 6785 - 6795.

[16] K. K. Chakrahari, R. P. B. Silalahi, T.-H. Chiu, X. Wang, N. Azrou, S. Kahlal, Y.-C. Liu, M.-H. Chiang, J.-Y. Saillard, and C. W. Liu, Angew. Chem. Int. Ed., 2019, 58, 4943-4947. 
[17] R. P. B. Silalahi, K. K. Chakrahari, J.-H. Liao, S. Kahlal, Y.-C. Liu, M.-H.

Chiang, J.-Y. Saillard, and C. W. Liu, Chem. Asian J. 2018, 13, 500-504.

[18] a) R. M. D. Calvi, D. H. Andrews, W. C. Lineberger, Chem.Phys. Lett., 2007,

442, 12-16; b) L. Andrews, X. Wang, J. Am. Chem. Soc., 2003, 125, 11751-11760.

a) $\quad\left[\mathrm{Cu}_{28}\left(\mathrm{H}_{15}\left\{\mathrm{~S}_{2} \mathrm{CNR}_{2}\right\}_{12}\right]\left(\mathrm{PF}_{6}\right)+10 \mathrm{HC}_{2} \mathrm{Ph} \frac{\mathrm{I} \mathrm{HF}}{30{ }^{\circ} \mathrm{C}^{\prime} 12 \mathrm{~h}}\left[\mathrm{Cu}_{15}\left(\mathrm{H}_{2}\right)_{2}\left\{\mathrm{~S}_{2} \mathrm{CNR}_{2}\right\}_{6}\left(\mathrm{C}_{2} \mathrm{Ph}\right)_{6}\right]\left(\mathrm{PF}_{6}\right)\right.$

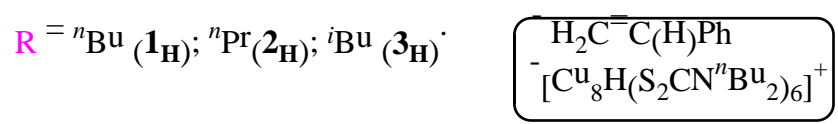

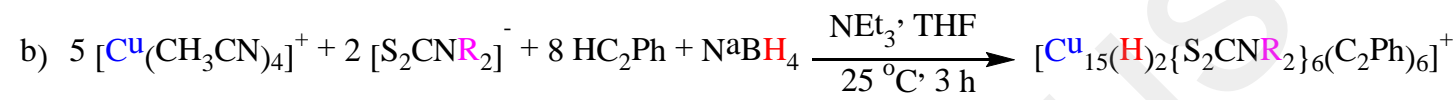

Scheme 1. Synthesis of clusters 1-3 from a) polyhydrido copper clusters, and b) mixing of substrates and borohydrides.

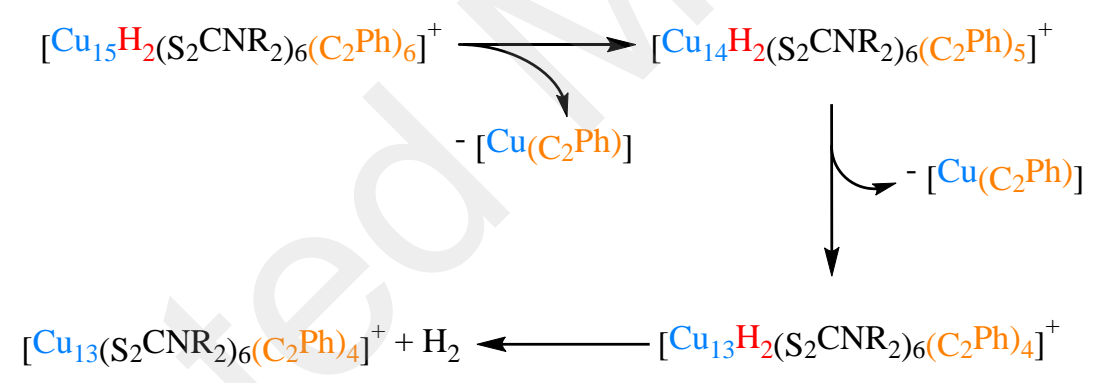

2-electron superatom

Scheme 2: Degradation of $\left[\mathrm{Cu}_{15}(\mathrm{H})_{2}\left\{\mathrm{~S}_{2} \mathrm{CNR}_{2}\right\}_{6}\left(\mathrm{C}_{2} \mathrm{Ph}\right)_{6}\right]^{+}$into a two electron superatom $\left[\mathrm{Cu}_{13}\left\{\mathrm{~S}_{2} \mathrm{CNR}_{2}\right\}_{6}\left(\mathrm{C}_{2} \mathrm{Ph}\right)_{4}\right]^{+}$.

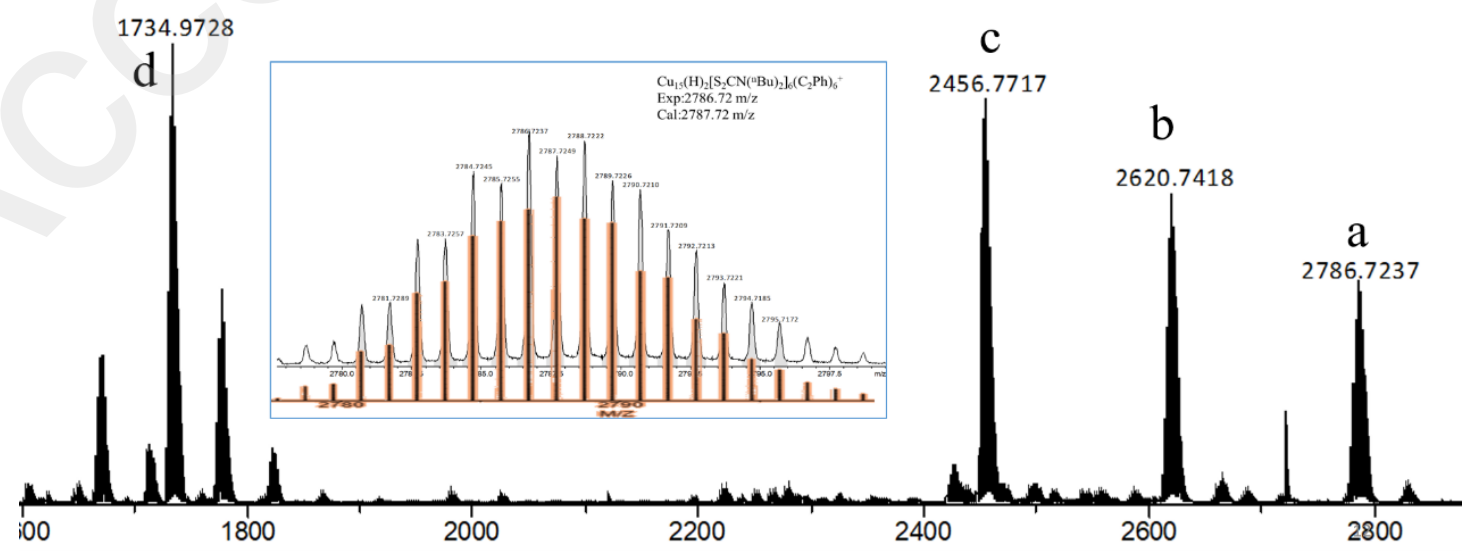


Figure 1. Positive mode ESI-MS of $\left[\mathbf{1}_{\mathbf{H}}\right]^{+}$(a) and fragment ion peaks $\left[\mathrm{Cu}_{14}(\mathrm{H})_{2}\left(\mathrm{~S}_{2} \mathrm{CN}^{n} \mathrm{Bu}_{2}\right)_{6}\left(\mathrm{C}_{2} \mathrm{Ph}\right)_{5}\right]^{+}(\mathrm{b}),\left[\mathrm{Cu}_{13}(\mathrm{H})_{2}\left(\mathrm{~S}_{2} \mathrm{CN}^{n} \mathrm{Bu}_{2}\right)_{6}\left(\mathrm{C}_{2} \mathrm{Ph}\right)_{4}\right]^{+}$(c), $\left[\mathrm{Cu}_{8} \mathrm{H}\left(\mathrm{S}_{2} \mathrm{CN}^{n} \mathrm{Bu}_{2}\right)_{6}\right]^{+}(\mathrm{d})$. Insets shows isotopic patterns for $\left[\mathbf{1}_{\mathbf{H}}\right]^{+}$, experimental on top and simulated on bottom.

a)

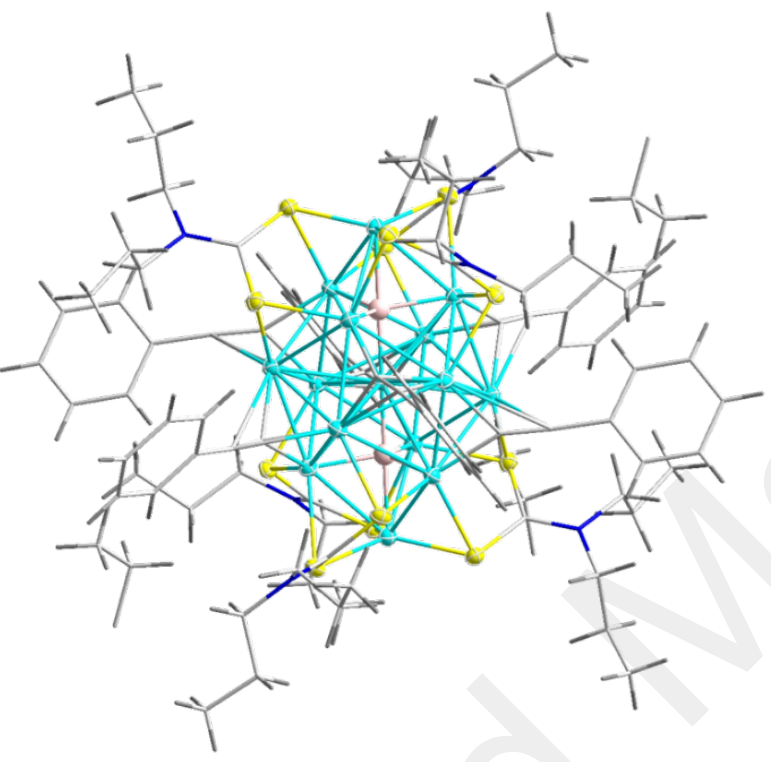

b)

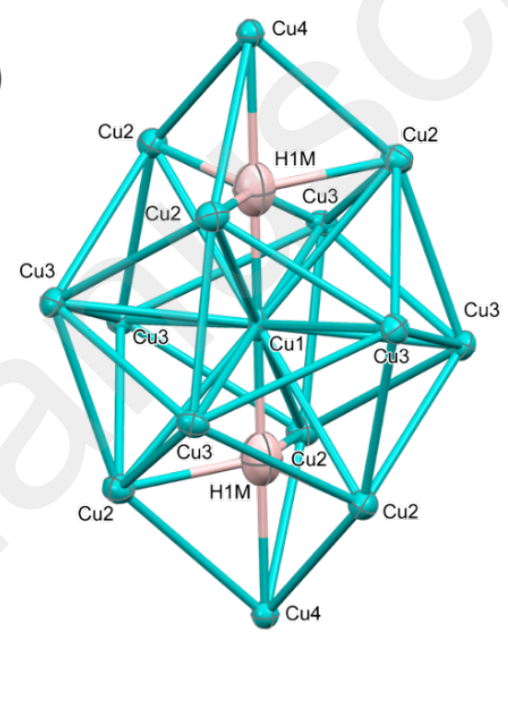

Figure 2. a) Structure of $\left[\mathrm{Cu}_{15}(\mathrm{H})_{2}\left\{\mathrm{~S}_{2} \mathrm{CN}^{n} \mathrm{Pr}_{2}\right\}_{6}\left(\mathrm{C}_{2} \mathrm{Ph}\right)_{6}\right]^{+}\left(2_{\mathbf{H}}\right)$ from neutron diffraction data. b). Side view of the bicapped icosahederal $\mathrm{Cu}_{14}$ shell encapsulating a copper dihydride unit. Color: green, copper; yellow, sulphur; blue, nitrogen; grey, carbon; pink, hydrogen. 


\section{Table of Contents}

A 15 nuclear copper dihydride cluster $\left[\mathrm{Cu}_{15}(\mathrm{H})_{2}\left(\mathrm{~S}_{2} \mathrm{CNR}_{2}\right)_{6}\left(\mathrm{C}_{2} \mathrm{Ph}\right)_{6}\right]\left(\mathrm{PF}_{6}\right)(\mathbf{1})$ containing a linear $\left[\mathrm{CuH}_{2}\right]^{-}$complex embeded in a $\left[\mathrm{Cu}_{14}\right]^{14+}$ cage was isolated and structurally characterized. This cluster readily converts to a two electron superatom cluster $\left[\mathrm{Cu}_{13}\right]^{11+}$ at elevated temperatures. Highly luminescent

$\left[\mathrm{AuCu}_{12}\left(\mathrm{~S}_{2} \mathrm{CNR}_{2}\right)_{6}\left(\mathrm{C}_{2} \mathrm{Ph}\right)_{4}\right]^{+}$cluster was isolated by recting 1 with $\mathrm{Au}(\mathrm{I})$ salts.

Copper-dihydride

Kiran Kumarvarma Chakrahari, Jingping Liao, Rhone P. Brocha Silalahi, Tzu-Hao Chiu, Jian-Hong Liao, Xiaoping Wang,* Samia Kahlal, Jean-Yves Saillard,* and C. W. Liu*

Isolation and Structural Elucidation of 15-nuclear Copper Dihydride Clusters: An Intermediate in the Formation of a 2-electron Copper Superatom

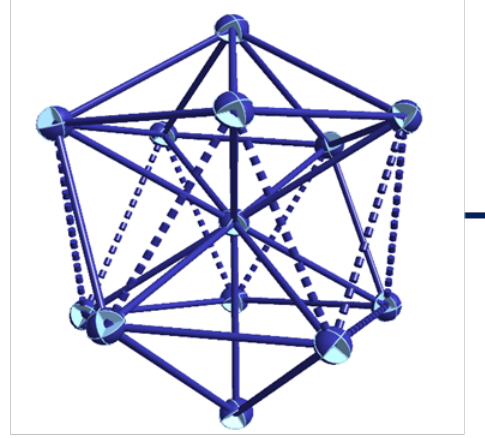

$\mathrm{Cu} @ \mathrm{Cu}_{12}($ Icosahedra $)$

$\left[\mathrm{Cu}_{15} \mathrm{H}_{2}\left(\mathrm{~S}_{2} \mathrm{CN}^{n} \mathrm{Pr}_{2}\right)_{6}\left(\mathrm{C}_{2} \mathrm{Ph}\right)_{6}\right] \mathrm{PF}_{6}$

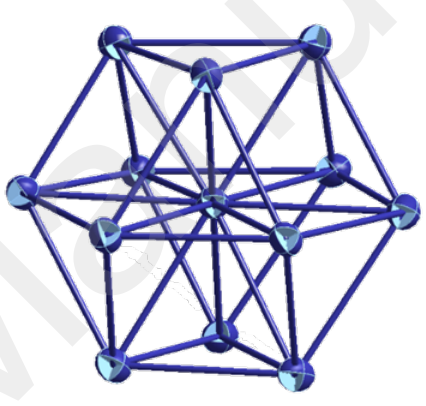

$\mathrm{Cu} @ \mathrm{Cu}_{12}($ Cuboctahedra $)$

$\left[\mathrm{Cu}_{13}\left(\mathrm{~S}_{2} \mathrm{CN}^{n} \mathrm{Pr}_{2}\right)_{6}\left(\mathrm{C}_{2} \mathrm{Ph}\right)_{4}\right] \mathrm{PF}_{6}$ 
Copyright WILEY-VCH Verlag GmbH \& Co. KGaA, 69469 Weinheim, Germany, 2018.

\section{Supporting Information}

\section{Isolation and Structural Elucidation of 15-nuclear Copper Dihydride Clusters: An Intermediate in the Formation of a 2-electron Copper Superatom}

Kiran Kumarvarma Chakrahari, Jingping Liao, Rhone P. Brocha Silalahi, Tzu-Hao

Chiu, Jian-Hong Liao, Xiaoping Wang, ${ }^{*}$ Samia Kahlal, Jean-Yves Saillard,* and C. W. Liu*

\section{EXPERIMENTAL SECTION}

Materials and Methods: All chemicals were purchased from commercial sources and used as received. All the reactions were conducted under an $\mathrm{Ar} / \mathrm{N}_{2}$ atmosphere using standard Schlenk techniques. $\left[\mathrm{Cu}_{28} \mathrm{H}_{15}\left\{\mathrm{~S}_{2} \mathrm{CNR}_{2}\right\}_{12}\right]\left(\mathrm{PF}_{6}\right), \quad\left[\mathrm{Cu}\left(\mathrm{CH}_{3} \mathrm{CN}\right)_{4}\right]\left(\mathrm{PF}_{6}\right)$, $\mathrm{K}\left(\mathrm{S}_{2} \mathrm{CNR}\right)$ ligands $\left(\mathrm{NR}=\mathrm{N}^{n} \mathrm{Pr}_{2}, \quad \mathrm{~N}^{n} \mathrm{Bu}_{2}\right.$ and $\left.\mathrm{N}^{i} \mathrm{Bu}_{2}\right)$, Triethylamine and phenylacetylene were prepared as described in the literature. ${ }^{[1-3]}$ Solvents were purified following standard protocols. Melting points were measured by using a Fargo MP-2D melting point apparatus. The elemental analyses were done using a Perkin-Elmer 2400 NCSH analyzer. NMR spectra were recorded on Bruker Advance DPX300 FT-NMR spectrometer that operates at $300 \mathrm{MHz}$ while recording ${ }^{1} \mathrm{H}, 121.5$ $\mathrm{MHz}$ for ${ }^{31} \mathrm{P}, 75.5 \mathrm{MHz}$ for ${ }^{13} \mathrm{C}$, and $46.1 \mathrm{MHz}$ for ${ }^{2} \mathrm{H}$. Residual solvent protons were used as a reference $\left(\delta, \mathrm{ppm}, \mathrm{CDCl}_{3}, 7.26\right) .{ }^{31} \mathrm{P} \mathrm{NMR}$ spectra were referenced to external 85\% $\mathrm{H}_{3} \mathrm{PO}_{4}$ at $\delta$ 0.00; Bruker Advance DPX400 FT-NMR spectrometer recording ${ }^{1} \mathrm{H}, 161.96 \mathrm{MHz}$ for ${ }^{31} \mathrm{P}, 100.6 \mathrm{MHz}$ for ${ }^{13} \mathrm{C}$, and $61.4 \mathrm{MHz}$ for ${ }^{2} \mathrm{H}$. Residual solvent protons were used as a reference (ppm, $\left.\mathrm{CDCl}_{3}, \delta=7.26\right) .{ }^{31} \mathrm{P}$ NMR spectra were referenced to external $85 \% \mathrm{H}_{3} \mathrm{PO}_{4}$ at $\delta 0.00$. ESI-mass spectra were recorded on a Bruker maXis ESI-QTOF (quadrupole time-of-flight) tandem mass spectrometer. UV-Visible absorption spectra were measured on a Perkin Elmer Lambda 750 spectrophotometer using quartz cells with path length of $1 \mathrm{~cm}$.

\section{Synthesis of $\left[\mathrm{Cu}_{15}(\mathrm{H})_{\mathbf{2}}\left(\mathrm{S}_{\mathbf{2}} \mathrm{CN}^{\mathrm{n}} \mathrm{Bu}_{2}\right)_{\mathbf{6}}\left(\mathrm{C}_{\mathbf{2}} \mathrm{Ph}\right)_{6}\right]^{+}$from $\left[\mathrm{Cu}_{28}(\mathrm{H})_{15}\left(\mathrm{~S}_{\mathbf{2}} \mathrm{CN}^{\mathrm{n}} \mathrm{Bu}_{2}\right)_{12}\right]^{+}$}

In a Flame-dried Schlenk tube, $\left[\mathrm{Cu}_{28}(\mathrm{H})_{15}\left(\mathrm{~S}_{2} \mathrm{CN}^{n} \mathrm{Bu}_{2}\right)_{12}\right]\left(\mathrm{PF}_{6}\right),(0.1 \mathrm{~g}, 0.022 \mathrm{mmol})$ was suspended in THF $(5 \mathrm{~mL})$ along with phenylacetylene $(24 \mu \mathrm{L}, 0.22 \mathrm{mmol})$ resulting mixture was stirred at $30{ }^{\circ} \mathrm{C}$ for $12 \mathrm{~h}$. The solvent was evaporated under vacuum and the residue was washed with diethyl ether $(3 \times 15 \mathrm{~mL})$ to remove styrene, followed by methanol $(3 \times 5 \mathrm{~mL})$ to remove $\left[\mathrm{Cu}_{8}(\mathrm{H})\left(\mathrm{S}_{2} \mathrm{CN}^{n} \mathrm{Bu}_{2}\right)_{6}\right]^{+}$and other ligand impurities. The residue was extracted in dichloromethane and filtered through 
aluminum oxide. Finally solvent was evaporated to dryness under vacuum to get a pure orange red powdered $\left[\mathrm{Cu}_{15} \mathrm{H}_{2}\left(\mathrm{~S}_{2} \mathrm{CN}^{n} \mathrm{Bu}_{2}\right)_{6}(\mathrm{C} \equiv \mathrm{CPh})_{6}\right]\left(\mathrm{PF}_{6}\right)\left(\mathbf{1}_{\mathbf{H}}\right)(0.035 \mathrm{~g}, 54 \%$, based on $\mathbf{C u}$ ). Similarly, deuterium analogue $\left(\mathbf{1}_{\mathbf{D}}\right)$ was synthesized by reacting $\left[\mathrm{Cu}_{28}(\mathrm{D})_{15}\left(\mathrm{~S}_{2} \mathrm{CN}^{n} \mathrm{Bu}_{2}\right)_{12}\right]\left(\mathrm{PF}_{6}\right)$, (0.1 g, $\left.0.022 \mathrm{mmol}\right)$, phenylacetylene (24 $\mu \mathrm{L}, 0.22$ mmol) resulted in the formation of $\left[\mathrm{Cu}_{15}(\mathrm{D})_{2}\left(\mathrm{~S}_{2} \mathrm{CN}^{n} \mathrm{Bu}_{2}\right)_{6}(\mathrm{C} \equiv \mathrm{CPh})_{6}\right]\left(\mathrm{PF}_{6}\right)\left(\mathbf{1}_{\mathbf{D}}\right),(0.03$ g, $46 \%$, based on $\mathrm{Cu}$ ) along with mono deuterated-styrene and $\left[\mathrm{Cu}_{8}(\mathrm{D})\left(\mathrm{S}_{2} \mathrm{CN}^{n} \mathrm{Bu}_{2}\right)_{6}\right]^{+}$ as byproducts.

\section{Synthesis of $\left[\mathrm{Cu}_{15}(\mathrm{H})_{2}\left(\mathrm{~S}_{2} \mathrm{CN}^{n} \mathrm{Bu}_{2}\right)_{6}(\mathrm{C} \equiv \mathrm{CPh})_{6}\right]\left(\mathrm{PF}_{6}\right)$ from $\mathrm{Cu}(\mathrm{I})$.}

In a Flame-dried Schlenk tube, $\mathrm{K}\left(\mathrm{S}_{2} \mathrm{CN}^{\mathrm{n}} \mathrm{Bu}_{2}\right) \quad(0.0486 \mathrm{~g}, \quad 0.2 \mathrm{mmol})$ and phenylacetylene $(80 \mu \mathrm{L}, 0.8 \mathrm{mmol})$ was suspended in THF $(30 \mathrm{~mL})$ addtion $\left[\mathrm{Cu}\left(\mathrm{CH}_{3} \mathrm{CN}\right)_{4}\right]\left(\mathrm{PF}_{6}\right)(0.1863 \mathrm{~g}, 0.5 \mathrm{mmol})$ and Triethylamine (80 $\left.\mu \mathrm{L}, 0.8 \mathrm{mmol}\right)$ reation 5 minutes, addtional $\mathrm{NaBH}_{4}(0.0037 \mathrm{~g}, 0.1 \mathrm{mmol})$ the resulting mixture was stirred at room temperature for $3 \mathrm{hr}$. The solvent was evaporated under vacuum and residue was dissolved in DCM and washed with water $(3 \times 15 \mathrm{~mL})$. After separation of organic layer, theorganic layer was passed through $\mathrm{Al}_{2} \mathrm{O}_{3}$, Finally the $\mathrm{Al}_{2} \mathrm{O}_{3}$ red solid was dissolved with Acetone, and solvent was evaporated under vacuum to get a orange red powdered for $\left[\mathrm{Cu}_{15}(\mathrm{H})_{2}\left(\mathrm{~S}_{2} \mathrm{CN}^{\mathrm{n}} \mathrm{Bu}_{2}\right)_{6}(\mathrm{C} \equiv \mathrm{CPh})_{6}\right]\left(\mathrm{PF}_{6}\right)\left(\mathbf{1}_{\mathbf{H}}\right),(0.068 \mathrm{~g}, 70 \%$, based on $\mathrm{Cu}$ ), Mp: $242{ }^{\circ} \mathrm{C}$ (dec.). The deuterium analog (1, $)$ was synthesized by reacting $\mathrm{NaBD}_{4} \quad(0.0042 \mathrm{~g}, \quad 0.1 \quad \mathrm{mmol}), \quad$ formation of $\left[\mathrm{Cu}_{15}(\mathrm{D})_{2}\left(\mathrm{~S}_{2} \mathrm{CN}^{n} \mathrm{Bu}_{2}\right)_{6}(\mathrm{C} \equiv \mathrm{CPh})_{6}\right]\left(\mathrm{PF}_{6}\right)\left(\mathbf{1}_{\mathbf{D}}\right)(0.070 \mathrm{~g}, 73 \%$, based on $\mathrm{Cu}), \mathrm{Mp}: 242{ }^{\circ} \mathrm{C}$ (dec.).

(1 $\left.\mathbf{1}_{\mathbf{H}}\right)$ : ESI-MS: $\mathrm{m} / \mathrm{z}$ (Cal. 2786.7) $2787.7\left(\mathrm{M}^{+}\right)$for $\left[\mathrm{Cu}_{15}(\mathrm{H})_{2}\left(\mathrm{~S}_{2} \mathrm{CN}^{n} \mathrm{Bu}_{2}\right)_{6}\left(\mathrm{C}_{2} \mathrm{Ph}\right)_{6}\right]\left(\mathrm{PF}_{6}\right)$ (1 $\mathbf{1}_{\mathbf{H}}{ }^{1} \mathrm{H}$ NMR (300 MHz, $\left.\mathrm{CDCl}_{3}, \mathrm{ppm}\right): 7.12 \sim 7.45$ (m, 30H, $-C_{2} \mathrm{Ph}$ ), 5.07 (bs, 2H, $\mu_{5^{-}}$ $H$ ), $3.89 \sim 4.01$ (m, 24H, $\left.C H_{2}\right), 1.52 \sim 1.76\left(\mathrm{~m}, 24 \mathrm{H}, C_{2}\right), 1.11 \sim 1.32\left(\mathrm{~m}, 24 \mathrm{H}, C_{2}\right)$, $0.68 \sim 0.86$ (d, 36H, $\left.\mathrm{CH}_{3}\right) ;{ }^{13} \mathrm{C} \mathrm{NMR}\left(75.5 \mathrm{MHz}, \mathrm{CDCl}_{3}, \mathrm{ppm}\right): 202.30,132.11,128.44$, 127.46, 125.18, 49.35, 29.14, 20.04, 13.73; ${ }^{31} \mathrm{P}\left\{{ }^{1} \mathrm{H}\right\}$ NMR (121.49 MHz, $\mathrm{CDCl}_{3}$, ppm): -143.11 ( $\left.{ }^{1} J_{\mathrm{PF}}=714 \mathrm{~Hz}\right) .\left(\mathbf{1}_{\mathbf{H}}\right)$ Elem. Anal: (Cal. C\% 41.77; H\% 4.81; N\% 2.87; S\% 13.12). Found: C\% 40.91; H\% 4.73; N\% 2.96; S\% 12.95.

(1 $\left.\mathbf{1}_{\mathbf{D}}\right)$ : ESI-MS: $\mathrm{m} / \mathrm{z}$ (Cal. 2789.7) $2788.7\left(\mathrm{M}^{+}\right)$for $\left[\mathrm{Cu}_{15}(\mathrm{D})_{2}\left(\mathrm{~S}_{2} \mathrm{CN}^{n} \mathrm{Bu}_{2}\right)_{6}\left(\mathrm{C}_{2} \mathrm{Ph}\right)_{6}\right]\left(\mathrm{PF}_{6}\right)$ (1 D $:{ }^{1} \mathrm{H}$ NMR (300 MHz, $\left.\mathrm{CDCl}_{3}, \mathrm{ppm}\right): 7.12 \sim 7.45$ (m, 30H, $\left.-C_{2} P h\right), 3.89 \sim 4.01$ (m, 24H, $\mathrm{CH}_{2}$ ), 1.52 1.76 (m, 24H, $\mathrm{CH}_{2}$ ), $1.11 \sim 1.32$ (m, 24H, $\left.\mathrm{CH}_{2}\right), 0.68 \sim 0.86$ (d, 36H, $\left.\mathrm{CH}_{3}\right) \mathrm{ppm} ;{ }^{31} \mathrm{P}\left\{{ }^{1} \mathrm{H}\right\}$ NMR (121.49 MHz, $\left.\mathrm{CDCl}_{3}, \mathrm{ppm}\right):-143.11\left({ }^{1} J_{\mathrm{PF}}=714 \mathrm{~Hz}\right) ;{ }^{2} \mathrm{H}$ NMR (46.1 MHz, $\left.\mathrm{CDCl}_{3}, \mathrm{ppm}\right): 4.98$ (bs, 2D, $\left.\mu_{5}-\mathrm{D}\right)$.

\section{Synthesis of $\left[\mathrm{Cu}_{15}(\mathrm{H})_{2}\left(\mathrm{~S}_{2} \mathrm{CN}^{\mathrm{n}} \mathrm{Pr}_{2}\right)_{6}(\mathrm{C} \equiv \mathrm{CPh})_{6}\right]\left(\mathrm{PF}_{6}\right)$ from $\mathrm{Cu}(\mathrm{I})$.}

In a Flame-dried Schlenk tube, $\mathrm{K}\left(\mathrm{S}_{2} \mathrm{CN}^{n} \mathrm{Pr}_{2}\right) \quad(0.0432 \mathrm{~g}, \quad 0.2 \mathrm{mmol})$ and phenylacetylene $(80 \mu \mathrm{L}, 0.8 \mathrm{mmol})$ was suspended in THF (30 mL) addtion $\left[\mathrm{Cu}\left(\mathrm{CH}_{3} \mathrm{CN}\right)_{4}\right]\left(\mathrm{PF}_{6}\right)(0.1863 \mathrm{~g}, 0.5 \mathrm{mmol})$ and Triethylamine $(80 \mu \mathrm{L}, 0.8 \mathrm{mmol})$ 
reation 5 minute, addtional $\mathrm{NaBH}_{4}(0.0037 \mathrm{~g}, 0.1 \mathrm{mmol})$ the resulting mixture was stirred at room temperature for $3 \mathrm{hr}$. The solvent was evaporated under vacuum and residue was dissolved in DCM and washed with water $(3 \times 15 \mathrm{~mL})$. After separation of organic layer, theorganic layer was passed through $\mathrm{Al}_{2} \mathrm{O}_{3}$. Finally the $\mathrm{Al}_{2} \mathrm{O}_{3}$ red solid was dissolved with acetone, and solvent was evaporated under vacuum to get a orange red powdered for $\left[\mathrm{Cu}_{15}(\mathrm{H})_{2}\left(\mathrm{~S}_{2} \mathrm{CN}^{n} \mathrm{Pr}_{2}\right)_{6}(\mathrm{C} \equiv \mathrm{CPh})_{6}\right]\left(\mathrm{PF}_{6}\right)\left(\mathbf{2}_{\mathbf{H}}\right)$, $(0.057 \mathrm{~g}, 65 \%$, based on $\mathrm{Cu}$ ), Mp:185 ${ }^{\circ} \mathrm{C}$ (dec.). The deuterium analog (2 $\left.\mathbf{2}_{\mathbf{D}}\right)$ was synthesized by reacting $\mathrm{NaBD}_{4}(0.0042 \mathrm{~g}, 0.1 \mathrm{mmol})$, formation of $\left[\mathrm{Cu}_{15}(\mathrm{D})_{2}\left(\mathrm{~S}_{2} \mathrm{CN}^{n} \mathrm{Pr}_{2}\right)_{6}(\mathrm{C} \equiv \mathrm{CPh})_{6}\right]\left(\mathrm{PF}_{6}\right)\left(2_{\mathrm{D}}\right)$ (0.059 g, 65\%, based on $\mathrm{Cu}$ ), $\mathrm{Mp}: 185^{\circ} \mathrm{C}$ (dec.).

(2 $\left.\mathbf{H}_{\mathrm{H}}\right):$ ESI-MS:m/z (Cal. 2616.5) $2618.5\left(\mathrm{M}^{+}\right)$for $\left[\mathrm{Cu}_{15}(\mathrm{H})_{2}\left(\mathrm{~S}_{2} \mathrm{CN}^{n} \mathrm{Pr}_{2}\right)_{6}\left(\mathrm{C}_{2} \mathrm{Ph}\right)_{6}\right]\left(\mathrm{PF}_{6}\right)$ (2 ${ }_{\mathrm{H}}{ }^{1} \mathrm{H}$ NMR (300 MHz, $\left.\mathrm{CDCl}_{3}, \mathrm{ppm}\right): 7.14 \sim 7.49$ (m, 30H, $-\mathrm{C}_{2} \mathrm{Ph}$ ), 4.92 (bs, 2H, $\mu_{5^{-}}$ $H$ ), $3.67 \sim 4.03$ (t, 24H, $\mathrm{CH}_{2}$ ), 1.65 1.85 (m, 24H, $\mathrm{CH}_{2}$ ), 0.82 0.98 (d, 36H, $\mathrm{CH}_{3}$ ) ppm; ${ }^{13} \mathrm{C}$ NMR (75.5 MHz, $\left.\mathrm{CDCl}_{3}, \mathrm{ppm}\right):$ 203.12, 132.10, 131.83, 128.13, 128.07, 123.57, 59.48, 20.44, 11.126; ${ }^{31} \mathrm{P}\left\{{ }^{1} \mathrm{H}\right\}$ NMR (121.49 $\left.\mathrm{MHz}, \mathrm{CDCl}_{3}, \mathrm{ppm}\right):-143.11$ $\left({ }^{1} J_{\mathrm{PF}}=714 \mathrm{~Hz}\right)$. Elem. Anal: (Cal. C\% 39.10; H\% 4.23; N\% 3.04; S\% 13.91). Found: C\% 39.22; H\% 4.23; N\% 3.08.

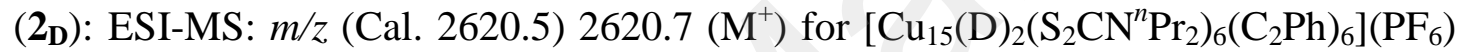
(2 ${ }_{\text {D }}{ }^{1} \mathrm{H}$ NMR (300 MHz, $\mathrm{CDCl}_{3}, \mathrm{ppm}$ ): $7.14 \sim 7.49$ (m, 30H, $-C_{2} \mathrm{Ph}$ ), 3.67 4.03 (t, 24H, $\mathrm{CH}_{2}$ ), $1.65 \sim 1.85$ (m, 24H, $\mathrm{CH}_{2}$ ), 0.82 0.98 (d, 36H, $\left.\mathrm{CH}_{3}\right) ;{ }^{2} \mathrm{H}$ NMR (46.1 MHz, $\left.\mathrm{CDCl}_{3}, \mathrm{ppm}\right): 5.04$ (bs, 2D, $\left.\mu_{5}-\mathrm{D}\right) ;{ }^{31} \mathrm{P}\left\{{ }^{1} \mathrm{H}\right\}$ NMR (121.49 $\left.\mathrm{MHz}, \mathrm{CDCl}_{3}, \mathrm{ppm}\right)$ : $143.11\left({ }^{1} J_{\mathrm{PF}}=714 \mathrm{~Hz}\right)$.

\section{Synthesis of $\left[\mathrm{Cu}_{15}(\mathrm{H})_{2}\left(\mathrm{~S}_{2} \mathrm{CN}^{i} \mathrm{Bu}_{2}\right)_{6}(\mathrm{C} \equiv \mathrm{CPh})_{6}\right]\left(\mathrm{PF}_{6}\right)$ from $\mathrm{Cu}(\mathrm{I})$.}

In a Flame-dried Schlenk tube, $\mathrm{K}\left(\mathrm{S}_{2} \mathrm{CN}^{i} \mathrm{Bu}_{2}\right)(0.0486 \mathrm{~g}, 0.2 \mathrm{mmol})$ and phenylacetylene (80 $\mu \mathrm{L}, 0.8 \mathrm{mmol})$ was suspended in THF (30 mL) addition $\left[\mathrm{Cu}\left(\mathrm{CH}_{3} \mathrm{CN}\right)_{4}\right]\left(\mathrm{PF}_{6}\right)(0.1863 \mathrm{~g}, 0.5 \mathrm{mmol})$ and Triethylamine $(80 \mu \mathrm{L}, 0.8 \mathrm{mmol})$ reaction 5 minutes, additional $\mathrm{NaBH}_{4}(0.0037 \mathrm{~g}, 0.1 \mathrm{mmol})$ the resulting mixture was stirred at room temperature for 3 hours. The solvent was evaporated under vacuum and residue was dissolved in DCM and washed with water $(3 \times 15 \mathrm{~mL})$. After separation of organic layer, theorganic layer was passed through $\mathrm{Al}_{2} \mathrm{O}_{3}$, finally the $\mathrm{Al}_{2} \mathrm{O}_{3}$ red solid was dissolved with acetone, and solvent was evaporated under vacuum to get a orange red powdered for $\left[\mathrm{Cu}_{15}(\mathrm{H})_{2}\left(\mathrm{~S}_{2} \mathrm{CN}^{i} \mathrm{Bu}_{2}\right)_{6}(\mathrm{C} \equiv \mathrm{CPh})_{6}\right]\left(\mathrm{PF}_{6}\right)\left(3_{\mathbf{H}}\right)$, (0.071 g, 74\%, based on $\mathrm{Cu}$ ), Mp: $235{ }^{\circ} \mathrm{C}$ (dec.). The deuterium analog $\left(\mathbf{3}_{\mathbf{D}}\right)$ was synthesized by reacting $\mathrm{NaBD}_{4}(0.0042 \mathrm{~g}, 0.1 \mathrm{mmol})$, formation of $\left[\mathrm{Cu}_{15}(\mathrm{D})_{2}\left(\mathrm{~S}_{2} \mathrm{CN}^{i} \mathrm{Bu}_{2}\right)_{6}(\mathrm{C} \equiv \mathrm{CPh})_{6}\right]\left(\mathrm{PF}_{6}\right)\left(3_{\mathbf{D}}\right) \quad(0.074 \mathrm{~g}, 75 \%$, based on $\mathrm{Cu}), \mathrm{Mp}: 235{ }^{\circ} \mathrm{C}$ (dec.).

(3H): ESI-MS: $m / z$ (Cal. 2786.7) $2787.7\left(\mathrm{M}^{+}\right)$for $\left[\mathrm{Cu}_{15}(\mathrm{H})_{2}\left(\mathrm{~S}_{2} \mathrm{CN}^{i} \mathrm{Bu}_{2}\right)_{6}\left(\mathrm{C}_{2} \mathrm{Ph}\right)_{6}\right]\left(\mathrm{PF}_{6}\right)$ (3H). ${ }^{1} \mathrm{H}$ NMR (300 MHz, $\left.\mathrm{CDCl}_{3}, \mathrm{ppm}\right): 7.12 \sim 7.45$ (m, 30H, $-\mathrm{C}_{2} \mathrm{Ph}$ ), 4.99 (bs, 2H, $\mu_{5}-$ $\mathrm{H}), 3.67 \sim 3.95$ (t, 24H, $\mathrm{CH}_{2}$ ), 2.31 2.49 (m, 12H, $\left.\mathrm{CH}\right), 0.68 \sim 0.97$ (d, $\left.36 \mathrm{H}, \mathrm{CH}_{3}\right) ;{ }^{13} \mathrm{C}$ 
NMR (75.5 MHz, $\mathrm{CDCl}_{3}$, ppm): 203.33, 132.12, 128.33, 127.98, 123.01, 65.74, 27.46, 20.04; ${ }^{31} \mathrm{P}\left\{{ }^{1} \mathrm{H}\right\}$ NMR (121.49 MHz, $\left.\mathrm{CDCl}_{3}, \mathrm{ppm}\right):-143.11\left({ }^{1} J_{\mathrm{PF}}=714 \mathrm{~Hz}\right)$. Elem. Anal: (Cal. C\% 41.77; H\% 4.81; N\% 2.87; S\% 13.12). Found: C\% 40.99; H\% 5.02; N\% 2.97; S\% 13.17.

(3 $)$ : ESI-MS: $m / z$ (Cal. 2789.7) $2788.7\left(\mathrm{M}^{+}\right)$for $\left[\mathrm{Cu}_{15}(\mathrm{D})_{2}\left(\mathrm{~S}_{2} \mathrm{CN}^{i} \mathrm{Bu}_{2}\right)_{6}\left(\mathrm{C}_{2} \mathrm{Ph}\right)_{6}\right]\left(\mathrm{PF}_{6}\right)$ (3 ${ }_{\mathbf{D}}{ }^{1} \mathrm{H}$ NMR (300 MHz, $\mathrm{CDCl}_{3}, \mathrm{ppm}$ ): 7.12 7.45 (m, 30H,- ${ }_{2} \mathrm{Ph}$ ), 3.67 3.95 (t, 24H, $\mathrm{CH}_{2}$ ), 2.31 2.49 (m, 12H, CH), 0.68 0.97 (d, 36H, $\left.\mathrm{CH}_{3}\right) ;{ }^{2} \mathrm{H}$ NMR (46.1 MHz, $\left.\mathrm{CDCl}_{3}, \mathrm{ppm}\right): 5.13$ (bs, 2D, $\left.\mu_{5}-\mathrm{D}\right)$.

\section{Synthesis of $\left[\mathrm{AuCu}_{12}\left(\mathrm{~S}_{2} \mathrm{CN}^{i} \mathrm{Bu}_{2}\right)_{\mathbf{6}}(\mathrm{C} \equiv \mathrm{CPh})_{4}\right]^{+}$from $\mathbf{3}_{\mathrm{H}}$ :}

Clusters $\mathbf{3}_{\mathbf{H}}(0.02 \mathrm{~g}, 0.007 \mathrm{mmol})$ and $\mathrm{Au}\left(\mathrm{PPh}_{3}\right) \mathrm{Cl}(0.003 \mathrm{~g}, 0.007 \mathrm{mmol})$ were dissolved in $2 \mathrm{~mL}$ of 2-methytetrahydrofuran solvent and the reaction mixture was stirred at $20{ }^{\circ} \mathrm{C}$ for one hour. The solvent was evaporated and the residue was subjected to alumina yielded $\left[\mathrm{AuCu}_{12}\left(\mathrm{~S}_{2} \mathrm{CN}^{i} \mathrm{Bu}_{2}\right)_{6}(\mathrm{C} \equiv \mathrm{CPh})_{4}\right]^{+}(0.01 \mathrm{~g}, 52 \%)$. ESIMS:m/z (Cal. 2588.79) 2588.84 for $\left[\mathrm{AuCu}_{12}\left(\mathrm{~S}_{2} \mathrm{CN}^{i} \mathrm{Bu}_{2}\right)_{6}\left(\mathrm{C}_{2} \mathrm{Ph}\right)_{4}\right]^{+} ;{ }^{1} \mathrm{H}$ NMR (300 $\left.\mathrm{MHz}, \mathrm{CDCl}_{3}, \mathrm{ppm}\right): 7.12 \sim 7.44\left(\mathrm{~m}, 20 \mathrm{H},-\mathrm{C}_{2} \mathrm{Ph}\right.$ ), 3.66 3.94 (t, 24H, $\mathrm{CH}_{2}$ ), 2.30 2.48 (m, 12H, $\mathrm{CH}$ ), 0.68 0.97 (d, 36H, $\mathrm{CH}_{3}$ ).

\section{X-ray Crystallography}

Single crystals suitable for X-ray diffraction analysis of $\mathbf{1}_{\mathbf{H}}, \mathbf{2}_{\mathbf{H}}$ and $\mathbf{3}_{\mathbf{H}}$ were obtained by slowly diffusing hexane into a concentrated acetone solution at $-5{ }^{\circ} \mathrm{C}$ temperature. The single crystals were mounted on the tip of glass fiber coated in paratone oil, then frozen at $150 \mathrm{~K}$. Data were collected on a Bruker APEX II CCD diffractometer using graphite monochromated Mo Ka radiation $(\lambda=0.71073 \AA$ ). Data reduction was performed with SAINT, ${ }^{[4]}$ Absorption corrections for the area detector were performed by using the SADABS program. ${ }^{[5]}$ The structure was solved by direct methods and refined by least-squares against $F^{2}$ using the SHELXL-2018/3 package, ${ }^{[6]}$ Incorporated in SHELXTL/PC V6.14. ${ }^{[7]}$

\section{Single Crystal Neutron Diffraction Experiment}

The location of hydrides in $\mathbf{2}_{\mathbf{H}}$ was confirmed by single crystal neutron diffraction experiment using the TOPAZ single-crystal neutron time-of-flight (TOF) Laue diffractometer at ORNL's Spallation Neutron Source. ${ }^{[8]}$ A reddish plate-shaped crystal $(0.75 \times 0.60 \times 0.35 \mathrm{~mm})$ was attached to a MiTeGen loop using a perfluorinated grease (Krytox GPL 205) and cooled to $100 \mathrm{~K}$ for data collection. A total of 23 crystal orientations optimized with CrystalPlan software ${ }^{[9]}$ were used to ensure better than 95\% coverage of a hemisphere of reciprocal space. Each orientation was measured for approximately 6.5 hrs with $30 \mathrm{C}$ of proton charge at the beam power of 1.4 MW. Peak integration and data reduction were performed in accordance 
with previously reported procedures. ${ }^{[10]}$ A spherical absorption correction was applied with $\mu=0.1213+0.08834 \lambda \mathrm{mm}^{-1}$. The reduced data were saved in SHELX HKLF2 format in which the neutron wavelength for each reflection was recorded separately. Non-hydrogen atom positions in the X-ray structure of $\mathbf{2}_{\mathbf{H}}$ were used for initial refinement of the neutron structure. Hydrogen atoms on carbon atoms are placed using the riding model available in SHELXL2014 ${ }^{[11]}$ with the default C-H distances for neutrons; only their displacement parameters are refined. Hydrogen is a negative scatter for neutrons. The peak for missing hydrogen atom appears as negative densities or holes in the difference Fourier map of the neutron data. The hydride peak (the deepest hole of -5.64) was located at $1.78 \AA$ from $\mathrm{Cu} 1$ atom in the asymmetric unit of $\mathbf{2}_{\mathbf{H}}$. The final refinement with the hydride atom included converged to low residues and featureless difference Fourier maps (deepest hole of -0.65). Selected neutron crystallographic data are listed in Table S2.

\section{ESI MS measurements}

Mass spectra of all samples were recorded on a Bruker maXis ESI-QTOF quadrupole time-of-flight tandem mass spectrometer. Mass spectrometry was calibrated with a tuning mix. The operating parameters of the electrospray ionization interface (ESI) in positive mode were: capillary voltage, $4000 \mathrm{~V}$; end plate offset, 500 $\mathrm{V}$; dry gas temperature, $200{ }^{\circ} \mathrm{C}\left(\mathrm{N}_{2}\right)$; dry gas flow, $4 \mathrm{~L} / \mathrm{min}$; and nebulizer pressure, 0.2 bar. Nitrogen was used as the nebulizing gas. The mass range scanned from 50 $\mathrm{m} / \mathrm{z}$ to $10000 \mathrm{~m} / \mathrm{z}$.

\section{Computational details}

For the sake of substantial CPU time saving, the substituants $\mathrm{R}={ }^{n} \mathrm{Bu},{ }^{n} \mathrm{Pr},{ }^{i} \mathrm{Bu}$ in $\mathbf{1}_{\mathbf{H}}, \mathbf{2}_{\mathbf{H}}$ and $\mathbf{3}_{\mathbf{H}}$, respectively, were replaced by $\mathrm{H}$. Such a simplification has been proven not to significantly affect the structure, bonding and most of properties of the investigated compounds (see for example ref. 14 of the manuscript). The geometry optimizations were performed by DFT calculations with the Gaussian 16 package, ${ }^{[12]}$ using the PBE0 functional, ${ }^{[13]}$ and the all-electron Def2-TZVP set from EMSL Basis Set Exchange Library. ${ }^{[14]}$ All the optimized geometries were characterized as true minima on their potential energy surface by harmonic vibrational analysis. The Wiberg bond indices were computed with the NBO 6.0 program. ${ }^{[15]}$ The UV-visible transitions were calculated by means of TD-DFT calculations. ${ }^{[16]}$ Only singlet-singlet, i.e. spin-allowed, transitions were computed. The UV-visible spectra were simulated from the computed from the TD-DFT transitions and their oscillator strengths by using the SWizard program, ${ }^{[17]}$ each transition being associated with a Gaussian function of half-height width equal to $3000 \mathrm{~cm}^{-1}$. The compositions of the molecular orbitals were calculated using the AOMix program. ${ }^{[18]}$ 
Table S1. Crystalographic data for $\left[\mathrm{Cu}_{15}(\mathrm{H})_{2}\left\{\mathrm{~S}_{2} \mathrm{CNR}_{2}\right\}_{6}\left(\mathrm{C}_{2} \mathrm{Ph}\right)_{6}\right]\left(\mathrm{PF}_{6}\right), \mathrm{R}={ }^{n} \mathrm{Bu}$, $1_{\mathrm{H}} ;{ }^{n} \mathrm{Pr}, 2_{\mathrm{H}}$ (data collected at $150 \mathrm{~K}$ and $100 \mathrm{~K}$, respectively); ${ }^{i} \mathrm{Bu}, 3_{\mathrm{H}}$.

\begin{tabular}{|c|c|c|c|c|}
\hline & $\mathbf{1}_{\mathrm{H}}$ & $2_{H}$ & $2_{H}$ & $3_{H}$ \\
\hline CCDC number & 1981041 & 1981042 & 1981043 & 1981045 \\
\hline formuls & \multicolumn{4}{|c|}{$\mathrm{C}_{102} \mathrm{H}_{140} \mathrm{Cu}_{15} \mathrm{~F}_{6} \mathrm{~N}_{6} \mathrm{C}_{90} \mathrm{H}_{116} \mathrm{Cu}_{15} \mathrm{~F}_{6} \mathrm{~N}_{6} \mathrm{P} \mathrm{C}_{90}$} \\
\hline lominumd & $\mathrm{PS}_{12}$ & $\mathrm{~S}_{12}$ & $\mathrm{~S}_{12}$ & $\mathrm{PS}_{12}$ \\
\hline fw & 2932.98 & 2764.67 & 2764.67 & 2932.98 \\
\hline Crystal symmrtry & Triclinic & Trigonal & Trigonal & Triclinic \\
\hline space group & $P(-) 1$ & $R(-) 3$ & $R(-) 3$ & $P(-) 1$ \\
\hline $\mathrm{a}, \AA$ & 13.8757(6) & $16.5595(4)$ & $16.5411(6)$ & $13.6613(3)$ \\
\hline $\mathrm{b}, \AA$ & $14.0092(5)$ & $16.5595(4)$ & $16.5411(6)$ & 13.9755(3) \\
\hline c, $\AA$ & 15.3927(6) & $31.6951(8)$ & $31.4310(12)$ & $17.5675(4)$ \\
\hline$\alpha, \operatorname{deg}$ & 71.3606(7) & 90 & 90 & $68.7807(5)$ \\
\hline$\beta, \operatorname{deg}$ & $85.5309(7)$ & 90 & 90 & $86.6061(6)$ \\
\hline$\gamma, \operatorname{deg}$ & 81.6909(8) & 120 & 120 & 71.4007(6) \\
\hline$V, \AA^{3}$ & 2803.84(19) & $7526.9(4)$ & $7447.6(6)$ & 2957.34(11) \\
\hline$Z$ & 1 & 3 & 3 & 1 \\
\hline$T, \mathrm{~K}$ & $150(2)$ & $150(2)$ & $100(2)$ & $150(2)$ \\
\hline$\rho_{\text {calcd }}, \mathrm{g} / \mathrm{cm}^{3}$ & 1.737 & 1.830 & 1.849 & 1.647 \\
\hline$\mu, \mathrm{mm}^{-1}$ & 3.074 & 3.429 & 3.465 & 2.914 \\
\hline$\theta_{\max }$, deg. & 28.29 & 25.99 & 27.49 & 26.40 \\
\hline $\begin{array}{l}\text { Reflections } \\
\text { collected }\end{array}$ & 27259 & 18675 & 11539 & 18166 \\
\hline Independent & 13510 & 3283 & 3817 & 11656 \\
\hline reflections & $\left(R_{\mathrm{int}}=0.0125\right)$ & $\left(R_{\mathrm{int}}=0.0271\right)$ & $\left(R_{\mathrm{int}}=0.0304\right)$ & $\left(R_{\mathrm{int}}=0.0153\right)$ \\
\hline $\begin{array}{l}\text { restraints } \\
\text { parameters }\end{array}$ & 356 / 669 & $7 / 224$ & $0 / 201$ & 4 / 661 \\
\hline $\begin{array}{l}\text { Final } R \text { indices } \\
{\left[I>2 \sigma(I)^{a, b}\right]}\end{array}$ & $\begin{array}{l}R 1=0.0273 \\
w R 2=0.0743\end{array}$ & $\begin{array}{l}R 1=0.0193 \\
w R 2=0.0522\end{array}$ & $\begin{array}{l}R 1=0.0456 \\
w R 2=0.1339\end{array}$ & $\begin{array}{l}R 1=0.0268 \\
w R 2=0.0657\end{array}$ \\
\hline$R$ indices (all data) & $\begin{array}{l}R 1=0.0312 \\
w R 2=0.0773\end{array}$ & $\begin{array}{l}R 1=0.0230 \\
w R 2=0.0539\end{array}$ & $\begin{array}{l}R 1=0.0544, \\
w R 2=0.1425\end{array}$ & $\begin{array}{l}R 1=0.0351 \\
w R 2=0.0695\end{array}$ \\
\hline GOF & 1.041 & 1.061 & 1.083 & 1.037 \\
\hline$\Delta \rho_{\max }$, e $\AA^{-3}$ & 1.353 & 0.511 & 2.378 & 0.805 \\
\hline$\Delta \rho_{\min }$, e $\AA^{-3}$ & -0.622 & -0.285 & -0.462 & -0.659 \\
\hline
\end{tabular}


Table S2. Selected neutron crystallographic data of $\mathbf{2}_{\mathrm{N}}$.

\begin{tabular}{ll}
\hline CCDC number & 1980144 \\
Chemical formula & $\mathrm{C}_{90} \mathrm{H}_{116} \mathrm{Cu}_{15} \mathrm{~F}_{6} \mathrm{~N}_{6} \mathrm{PS}_{12}$ \\
$M_{\mathrm{r}}$ & 2764.67 \\
Crystal system, space group & Trigonal, $R \overline{3}$ \\
Temperature $(\mathrm{K})$ & $100(2)$ \\
$a(\AA)$ & $16.5308(15)$ \\
$b(\AA)$ & $16.5308(15)$ \\
$c(\AA)$ & $31.519(5)$ \\
$V\left(\AA^{3}\right)$ & $7459.2(18)$ \\
$Z$ & 3 \\
Radiation type & Neutrons, $\lambda=0.4-3.5 \AA$ \\
$\mu$ (mm $\left.{ }^{-1}\right)$ & $0.12130+0.08834 \lambda$ \\
Crystal size (mm $\left.{ }^{3}\right)$ & $0.75 \times 0.60 \times 0.35$ \\
Data collection & \\
Diffractometer & TOPAZ \\
$T_{\text {min }} T_{\text {max }}$ & $0.812,0.929$ \\
No. of measured, independent and & $7837,1351,1141$ \\
observed $[I>2 \sigma(I)]$ reflections & 0.120 \\
$R_{\text {int }}$ & $0.066,0.108,1.13$ \\
$R\left[F^{2}>2 \sigma\left(F^{2}\right)\right], w R\left(F^{2}\right), S$ & 1351 \\
No. of reflections & 316 \\
No. of parameters & $1.27,-0.63$ \\
$\Delta \rho_{\text {max }}, \Delta \rho_{\text {min }}\left(\right.$ fm $\left.\AA^{-3}\right)$ & SHELXL2018/3 (Sheldrick, 2015). \\
Computer programs: SNS PyDas, Mantidplot, & \\
& \\
&
\end{tabular}


Table S3. Selected X-ray and/or neutron bond lengths $(\AA)$ for $\mathbf{1}_{\mathbf{H}}, \mathbf{2}_{\mathbf{H}}, \mathbf{3}_{\mathbf{H}}$ and $\left[\mathrm{Pd}(\mathrm{H})_{2} \mathrm{Cu}_{14}\left\{\mathrm{~S}_{2} \mathrm{CN}^{n} \mathrm{Bu}_{2}\right\}_{6}\left(\mathrm{C}_{2} \mathrm{Ph}\right)_{6}\right]\left(\mathbf{1}_{\mathbf{H P d}}\right) .{ }^{[19]} \mathrm{DFT}-\mathrm{optimized}$ distances of 1' are also given, together with their corresponding Wiberg indices in brackets.

\begin{tabular}{|c|c|c|c|c|c|c|c|c|c|c|}
\hline & $\mathrm{M}_{\text {cent }}-\mathrm{Cu}_{\text {ico }}$ & $\mathrm{Cu}_{\text {ico }}-\mathrm{Cu}_{\text {ico }}$ & $\mathrm{Cu}_{\text {cap }}-\mathrm{Cu}_{\text {ico }}$ & $\mathrm{M}_{\text {cent }}-\mathrm{H}$ & $\mathbf{M}_{\text {cap } \ldots H}$ & $\mathbf{M}_{\text {ico } \ldots H}$ & $\mathrm{Cu}_{\text {ico }}-\mathrm{S}$ & $\mathrm{Cu}_{\text {cap }}-\mathrm{S}$ & $\mathrm{Cu}_{\text {ico }}-\mathrm{C}$ & $\mathrm{C} \equiv \mathrm{C}$ \\
\hline $\mathbf{1}_{\mathrm{H}}$ & $\begin{array}{l}2.6538(3)- \\
2.7629(3) \\
\text { av. } 2.7079(3)\end{array}$ & $\begin{array}{l}2.5963(4)-3.3130(4) \\
\text { av. } 2.8473(4)\end{array}$ & $\begin{array}{l}2.5824(4)- \\
2.6511(4) \\
\text { av. } 2.6160(4)\end{array}$ & $1.68(3)$ & $2.13(3)$ & $\begin{array}{l}1.87(3)-1.94(3) \\
\text { av. } 1.90(3)\end{array}$ & $\begin{array}{l}2.3273(5)- \\
2.4655(6) \\
\text { av. } 2.3771(6)\end{array}$ & $\begin{array}{l}2.2389(6)- \\
2.2613(6) \\
\text { av. } 2.2503(6)\end{array}$ & $\begin{array}{l}1.980(2)-2.103(2) \\
\text { av. } 2.033(2)\end{array}$ & $\begin{array}{l}1.219(3)-1.226(3) \\
\text { av. } 1.222(3)\end{array}$ \\
\hline $\begin{array}{l}\mathbf{2 H} \\
(150 \mathrm{~K})\end{array}$ & $\begin{array}{l}2.6821(2)- \\
2.7174(2) \\
\text { av. } 2.6997(2)\end{array}$ & $\begin{array}{l}2.6253(3)-3.2081(7) \\
\text { av. } 2.8384(4)\end{array}$ & $2.6058(4)$ & $1.84(3)$ & 1.99(3) & $1.858(2)$ & $\begin{array}{l}2.3056(5)- \\
2.4663(5) \\
\text { av. } 2.3834(5)\end{array}$ & $2.2593(5)$ & $\begin{array}{l}1.9901(19)- \\
2.0720(18) \\
\text { av. } 2.0238(19)\end{array}$ & $1.223(3)$ \\
\hline $\begin{array}{l}\mathbf{2}_{\mathrm{H}} \\
(100 \mathrm{~K})\end{array}$ & $\begin{array}{l}2.6742(5)- \\
2.7123(5) \\
\text { av. } 2.6932(5)\end{array}$ & $\begin{array}{l}2.6193(7)-3.201(1) \\
\text { av. } 2.832(1)\end{array}$ & $2.6013(8)$ & $1.79(13)$ & $2.03(13)$ & $1.858(14)$ & $\begin{array}{l}2.3201(12)- \\
2.4639(12) \\
\text { av. } 2.3808(12)\end{array}$ & $2.2592(11)$ & $\begin{array}{l}1.980(4)-2.053(4) \\
\text { av. } 2.006(4)\end{array}$ & $1.241(7)$ \\
\hline $\begin{array}{l}2_{N} \\
(100 K, \\
\text { neutron) }\end{array}$ & $\begin{array}{l}2.670(4)-2.720(4) \\
\text { av. } 2.695(4)\end{array}$ & $\begin{array}{l}2.624(6)-3.205(7) \\
\text { av. } 2.833(6)\end{array}$ & $2.612(7)$ & $1.78(2)$ & $2.06(2)$ & $1.863(5)$ & $\begin{array}{l}2.310(13)- \\
2.485(13) \\
\text { av. } 2.388(13)\end{array}$ & $2.271(11)$ & $\begin{array}{l}1.993(7)-2.069(7) \\
\text { av. } 2.024(7)\end{array}$ & $1.227(7)$ \\
\hline $3_{H}$ & $\begin{array}{l}2.6138(3)- \\
2.7183(3) \\
\text { av. } 2.6765(3)\end{array}$ & $\begin{array}{l}2.5622(4)-3.1484(6) \\
\text { av. } 2.8172(4)\end{array}$ & $\begin{array}{l}2.6733(5)- \\
2.7223(4) \\
\text { av. } 2.6998(5)\end{array}$ & $1.62(3)$ & $2.39(2)$ & $\begin{array}{l}1.81(3)-1.90(3) \\
\text { av. } 1.85(3)\end{array}$ & $\begin{array}{l}2.3402(7)- \\
2.4056(7) \\
\text { av. } 2.3680(7)\end{array}$ & $\begin{array}{l}2.2390(7)- \\
2.2582(8) \\
\text { av. } 2.2469(7)\end{array}$ & $\begin{array}{l}1.989(3)-2.083(3) \\
\text { av. } 2.030(3)\end{array}$ & $\begin{array}{l}1.212(4)-1.215(4) \\
\text { av. } 1.213(4)\end{array}$ \\
\hline 1' (DFT) & $\begin{array}{l}2.687-2.726 \\
\text { av. } 2.707[0.069]\end{array}$ & $\begin{array}{l}2.636-3.011 \\
\text { av. } 2.747[0.052]\end{array}$ & $\begin{array}{l}2.636-2.666 \\
\text { av. } 2.651 \\
{[0.069]}\end{array}$ & $\begin{array}{l}1.725 \\
{[0.164]}\end{array}$ & $\begin{array}{l}2.212 \\
{[0.040]}\end{array}$ & $\begin{array}{l}1.838-1.847 \\
\text { av. } 1.843[0.069]\end{array}$ & $\begin{array}{l}2.366-2.493 \\
\text { av. } 2.427[0.158]\end{array}$ & $\begin{array}{l}2.279-2.287 \\
\text { av. } 2.282[0.224]\end{array}$ & $\begin{array}{l}2.000-2.092 \\
\text { av. } 2.036[0.1873]\end{array}$ & $\begin{array}{l}1.231-1.235 \\
\text { av. } 1.233[2.602]\end{array}$ \\
\hline $\begin{array}{l}\mathbf{1}_{\mathrm{HPd}} \\
\text { (neutron } \\
\text { ) }\end{array}$ & $\begin{array}{l}2.694(7)-2.795(7) \\
\text { av. } 2.735(7)\end{array}$ & $\begin{array}{l}2.628(9)-3.324(8) \\
\text { av. } 2.877(9)\end{array}$ & $\begin{array}{l}2.555(10)- \\
2.610(10) \\
\text { av. } 2.585(10)\end{array}$ & $1.759(14)$ & $2.020(16)$ & $\begin{array}{l}1.875(19)- \\
1.930(18) \\
\text { av. } 1.910(18)\end{array}$ & $\begin{array}{l}2.30(2)-2.50(2) \\
\text { av. } 2.40(2)\end{array}$ & $\begin{array}{l}2.27(2)-2.28(2) \\
\text { av. } 2.28(2)\end{array}$ & $\begin{array}{l}1.980(11)- \\
2.110(11) \\
\text { av. } 2.039(11)\end{array}$ & $\begin{array}{l}1.217(12)-1.244(12) \\
\text { av. } 1.231(12)\end{array}$ \\
\hline
\end{tabular}




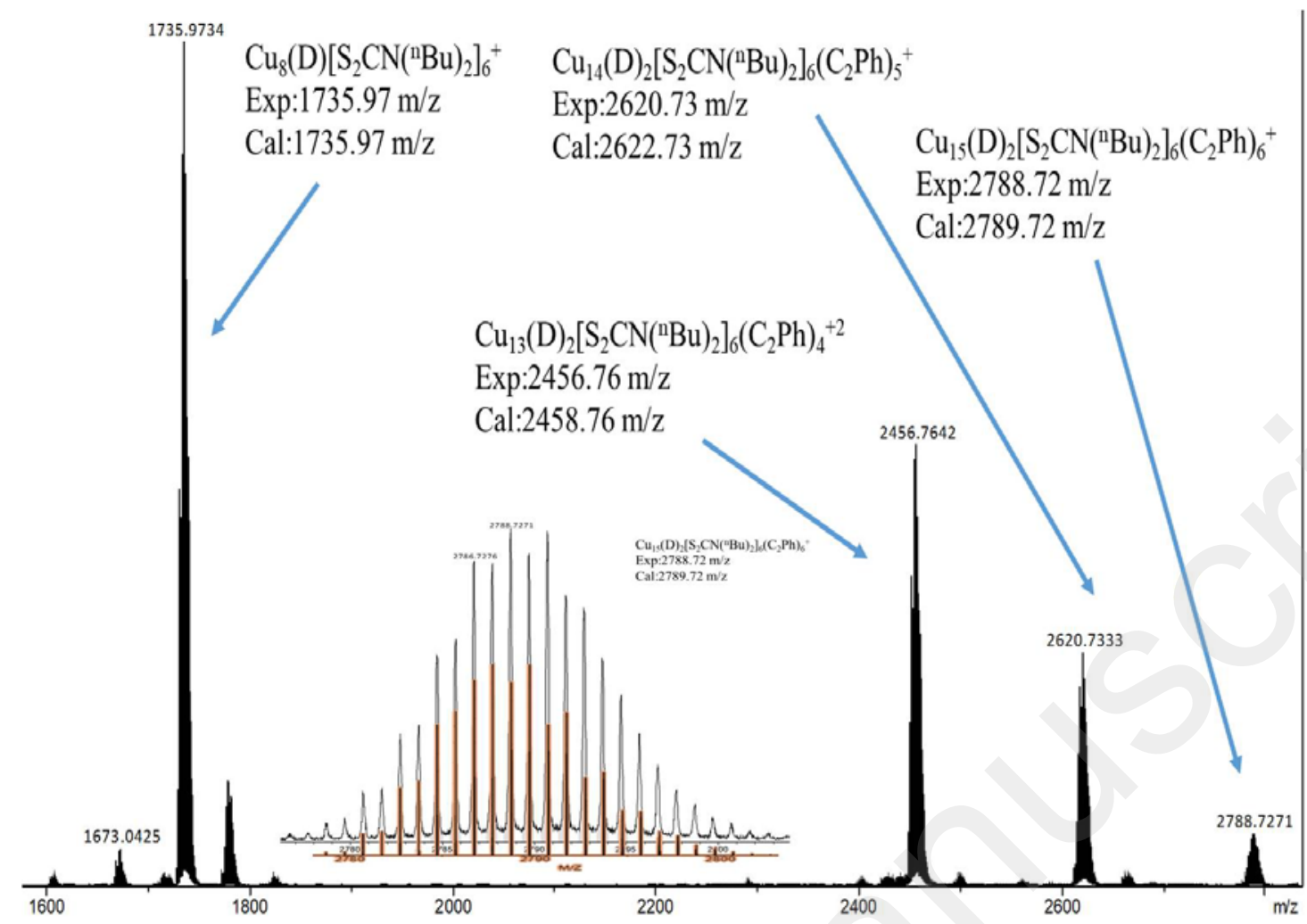

Figure S1. Positive ES-MS spectrum of $\left[\mathbf{1}_{\mathbf{D}}\right]^{+}$, the inset shows experimental one in the top and simulated one in the bottom. 


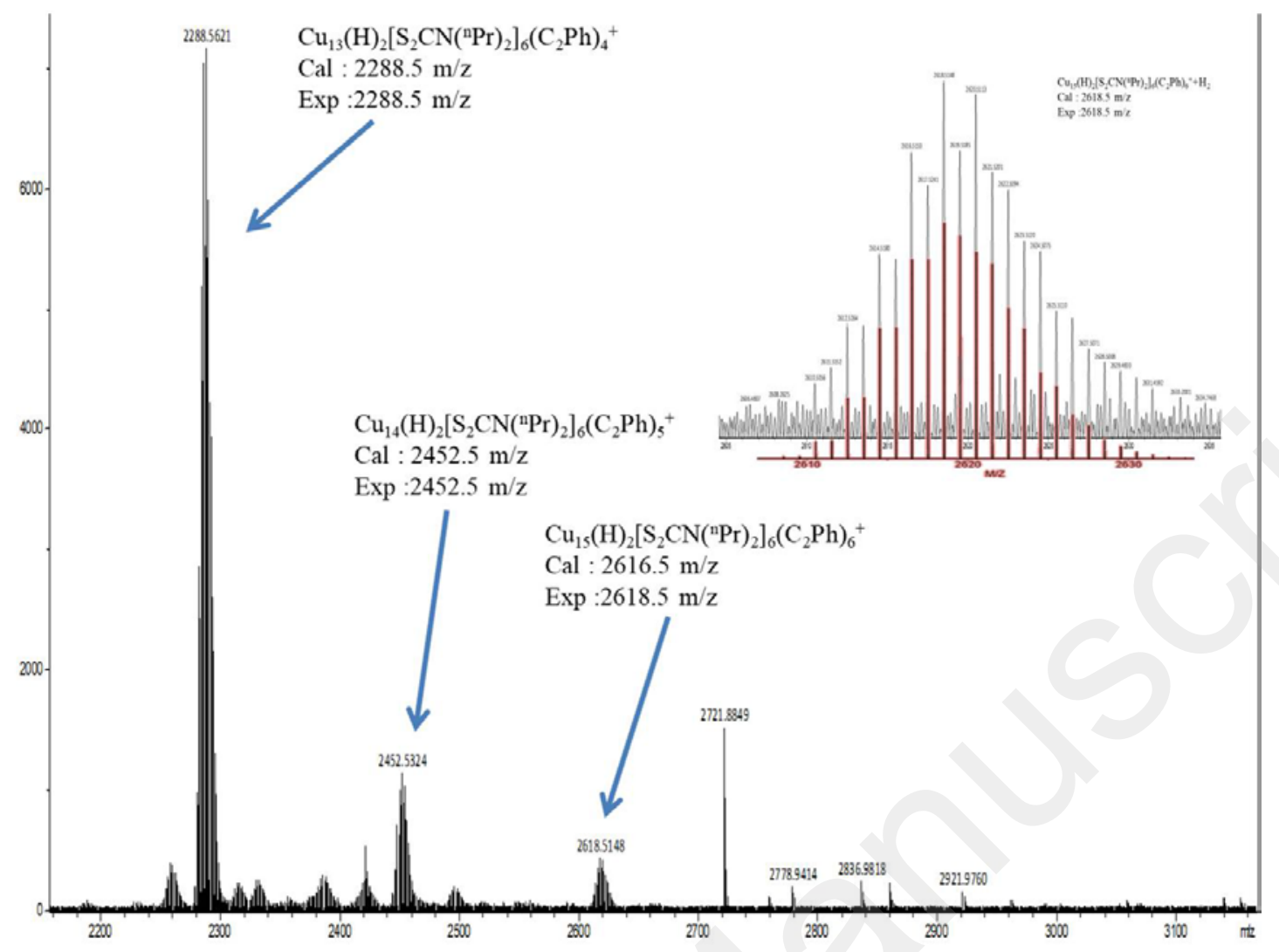

Figure S2. Positive ES-MS spectrum of $\left[\mathbf{2}_{\mathbf{H}}\right]^{+}$, the inset shows experimental one in the top and simulated one in the bottom. 


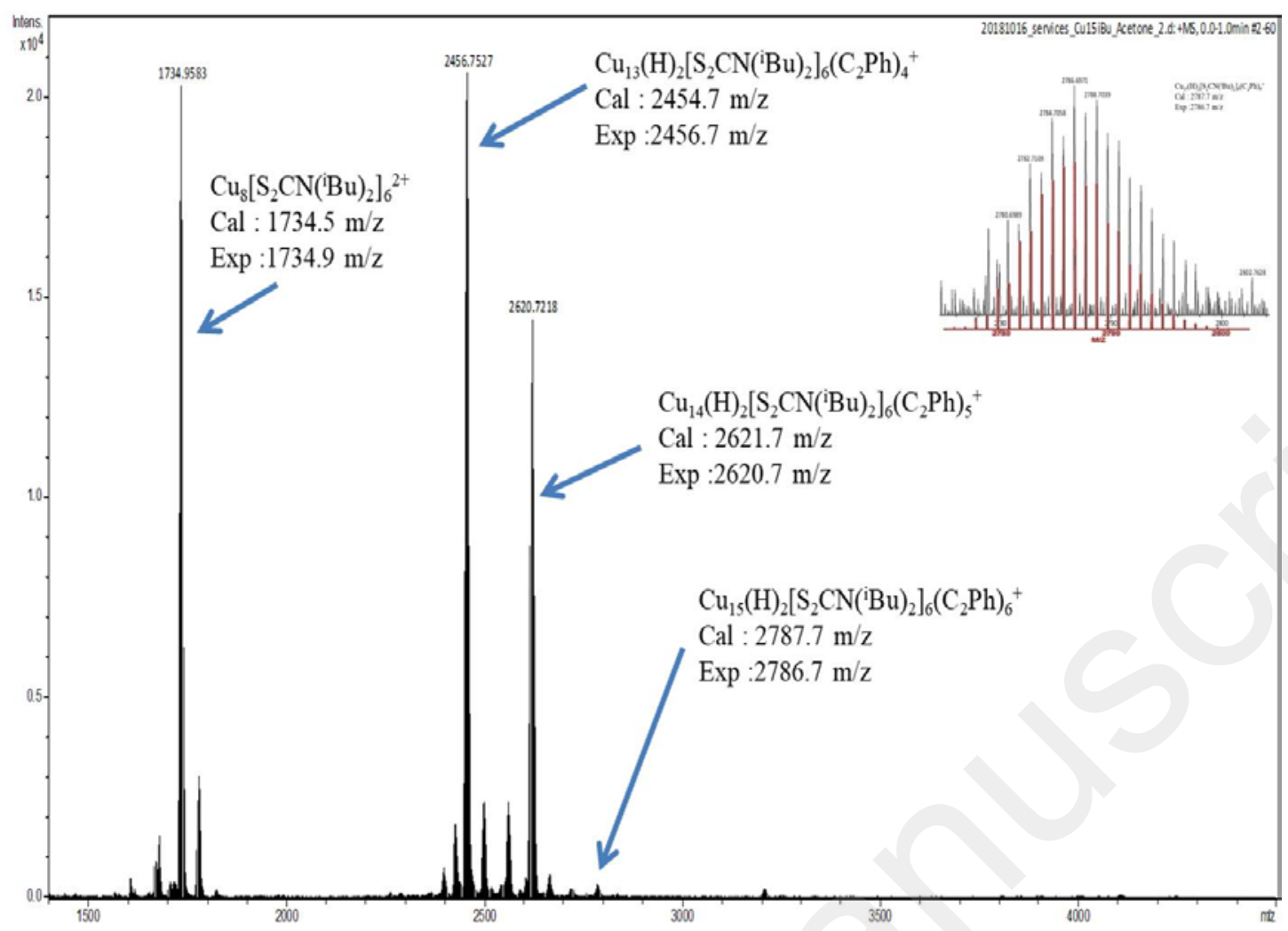

Figure S3. Positive ES-MS spectrum of $\left[3_{\mathbf{H}}\right]^{+}$, the inset shows experimental one in the top and simulated one in the bottom. 


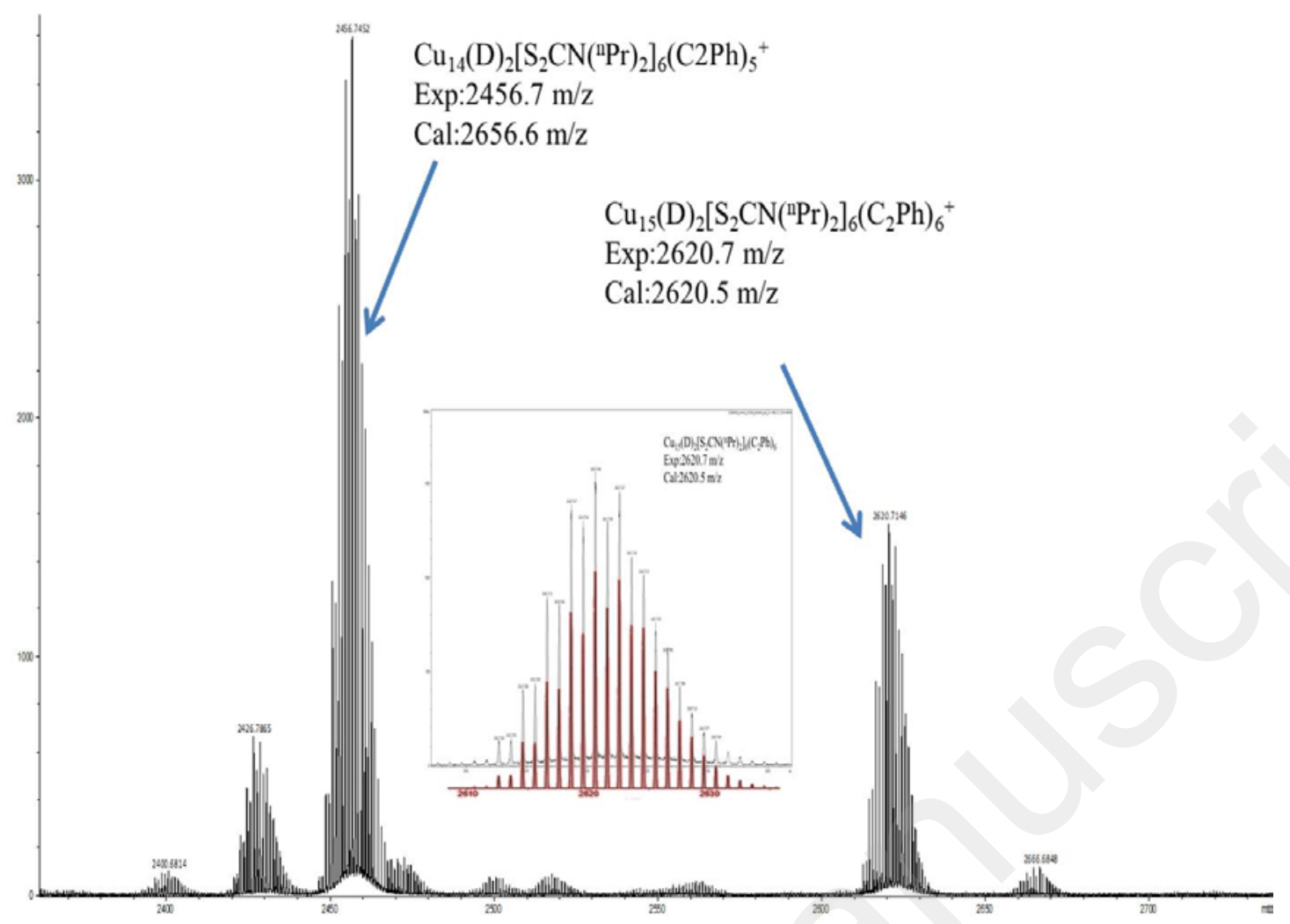

Figure S4. Positive ES-MS spectrum of $\left[\mathbf{2}_{\mathbf{D}}\right]^{+}$, the inset shows experimental one in the top and simulated one in the bottom. 


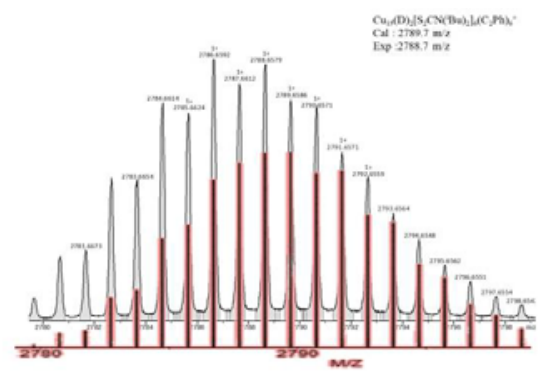

$\mathrm{Cu}_{15}(\mathrm{D})_{2}\left[\mathrm{~S}_{2} \mathrm{CN}\left({ }^{\mathrm{B}} \mathrm{Bu}\right)_{2}\right]_{6}\left(\mathrm{C}_{2} \mathrm{Ph}\right)_{6}{ }^{+}$

$\mathrm{Cal}: 2789.7 \mathrm{~m} / \mathrm{z}$

Exp : $2788.7 \mathrm{~m} / \mathrm{z}$

$\mathrm{Cu}_{14}(\mathrm{D})_{2}\left[\mathrm{~S}_{2} \mathrm{CN}\left({ }^{\mathrm{i}} \mathrm{Bu}\right)_{2}\right]_{6}\left(\mathrm{C}_{2} \mathrm{Ph}\right)_{5}{ }^{+}$

Cal : $2623.7 \mathrm{~m} / \mathrm{z}$

$\mathrm{Cu}_{13}(\mathrm{D})_{2}\left[\mathrm{~S}_{2} \mathrm{CN}\left({ }^{\mathrm{i}} \mathrm{Bu}\right)_{2}\right]_{6}\left(\mathrm{C}_{2} \mathrm{Ph}\right)_{4}{ }^{+} \operatorname{Exp}: 2620.7 \mathrm{~m} / \mathrm{z}$

$\mathrm{Cal}: 2458.7 \mathrm{~m} / \mathrm{z}$

$\operatorname{Exp}: 2456.7 \mathrm{~m} / \mathrm{z}$

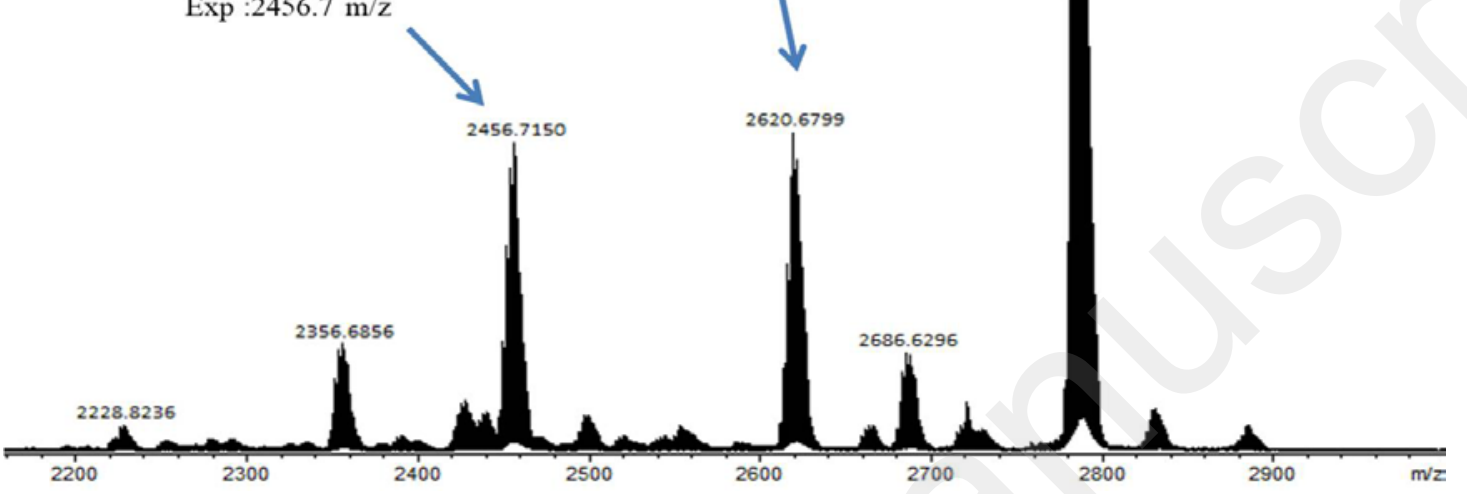

Figure S5. Positive ES-MS spectrum of $\left[\mathbf{3}_{\mathbf{D}}\right]^{+}$, the inset shows experimental one in the top and simulated one in the bottom. 
a.
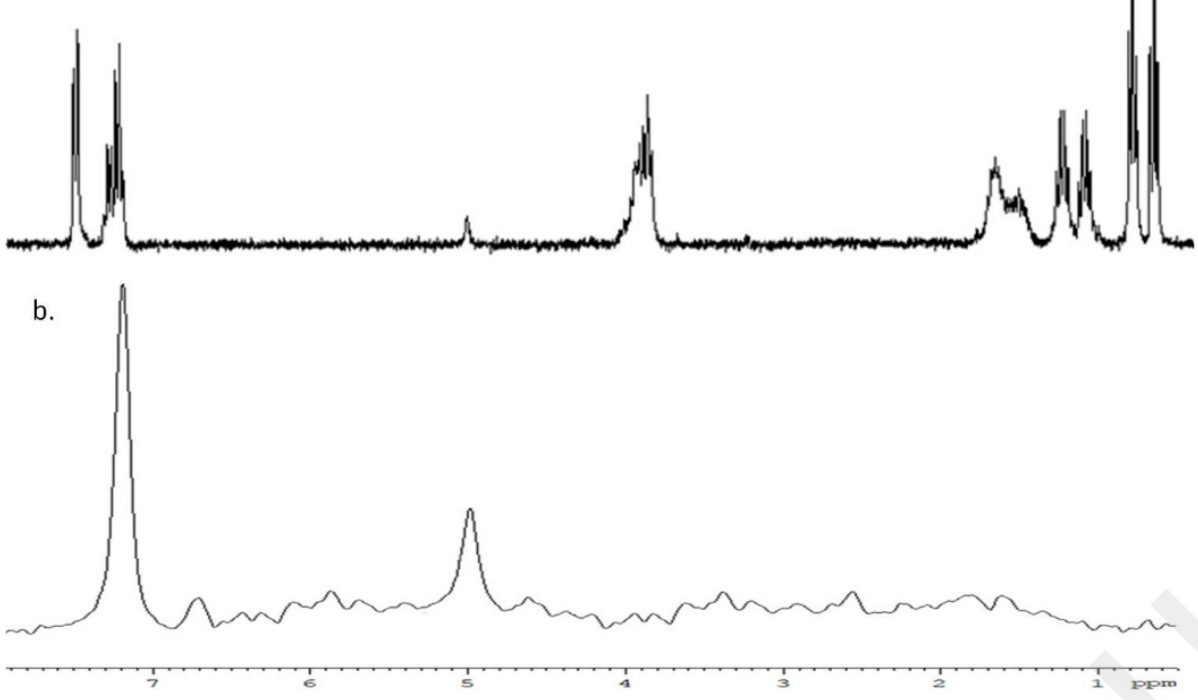

Figure S6. a) ${ }^{1} \mathrm{H}$ NMR spectrum of compound $\mathbf{1}_{\mathbf{H}}$ in $\mathrm{CDCl}_{3 ;}$ b) ${ }^{2} \mathrm{H}$ NMR spectrum of compound $\mathbf{1}_{\mathbf{D}}$ in $\mathrm{CHCl}_{3}$. 


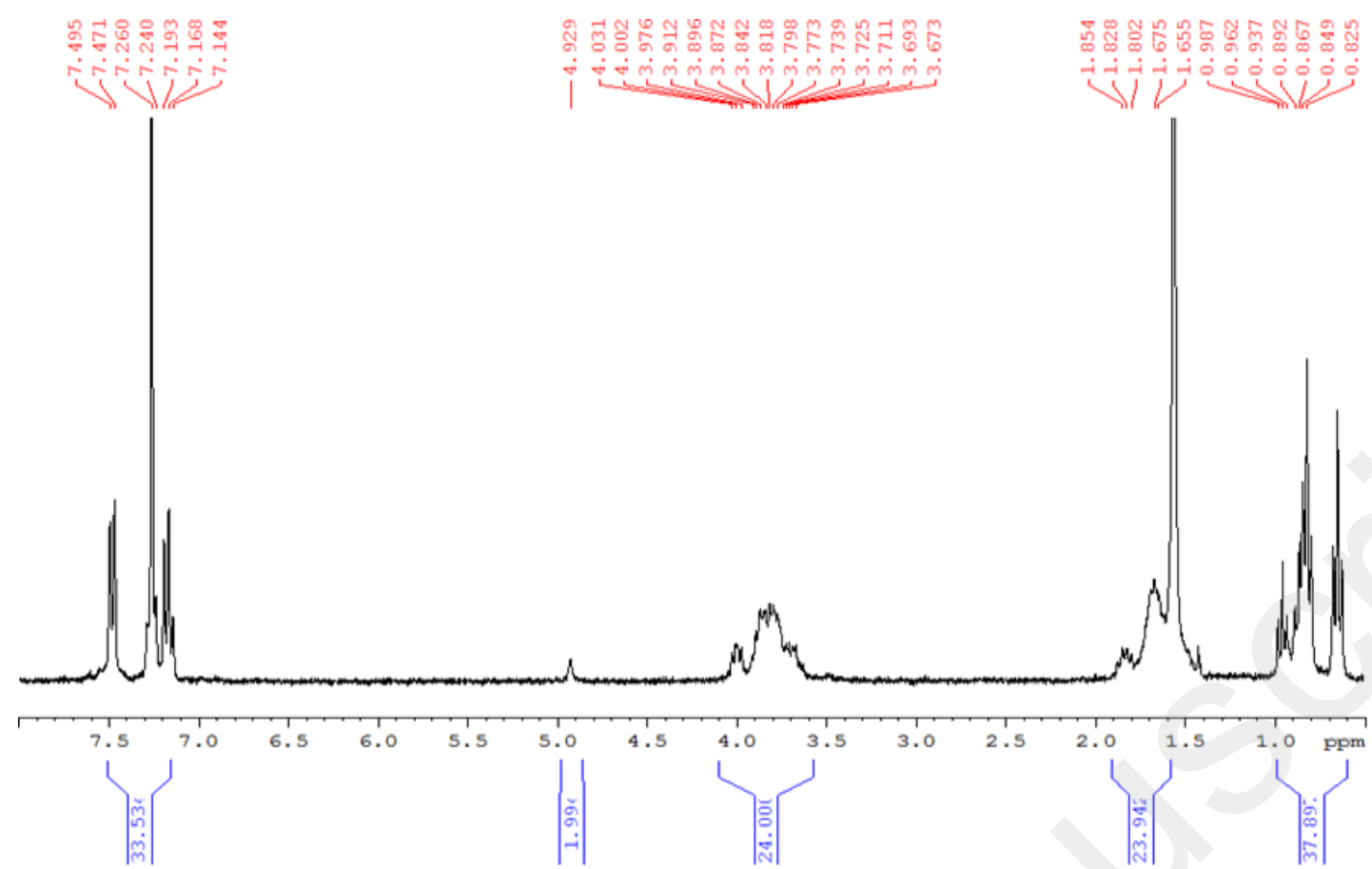

Figure S7. ${ }^{1} \mathrm{H}$ NMR spectrum of $\mathbf{2}_{\mathbf{H}}$ in $\mathrm{CDCl}_{3}$. 


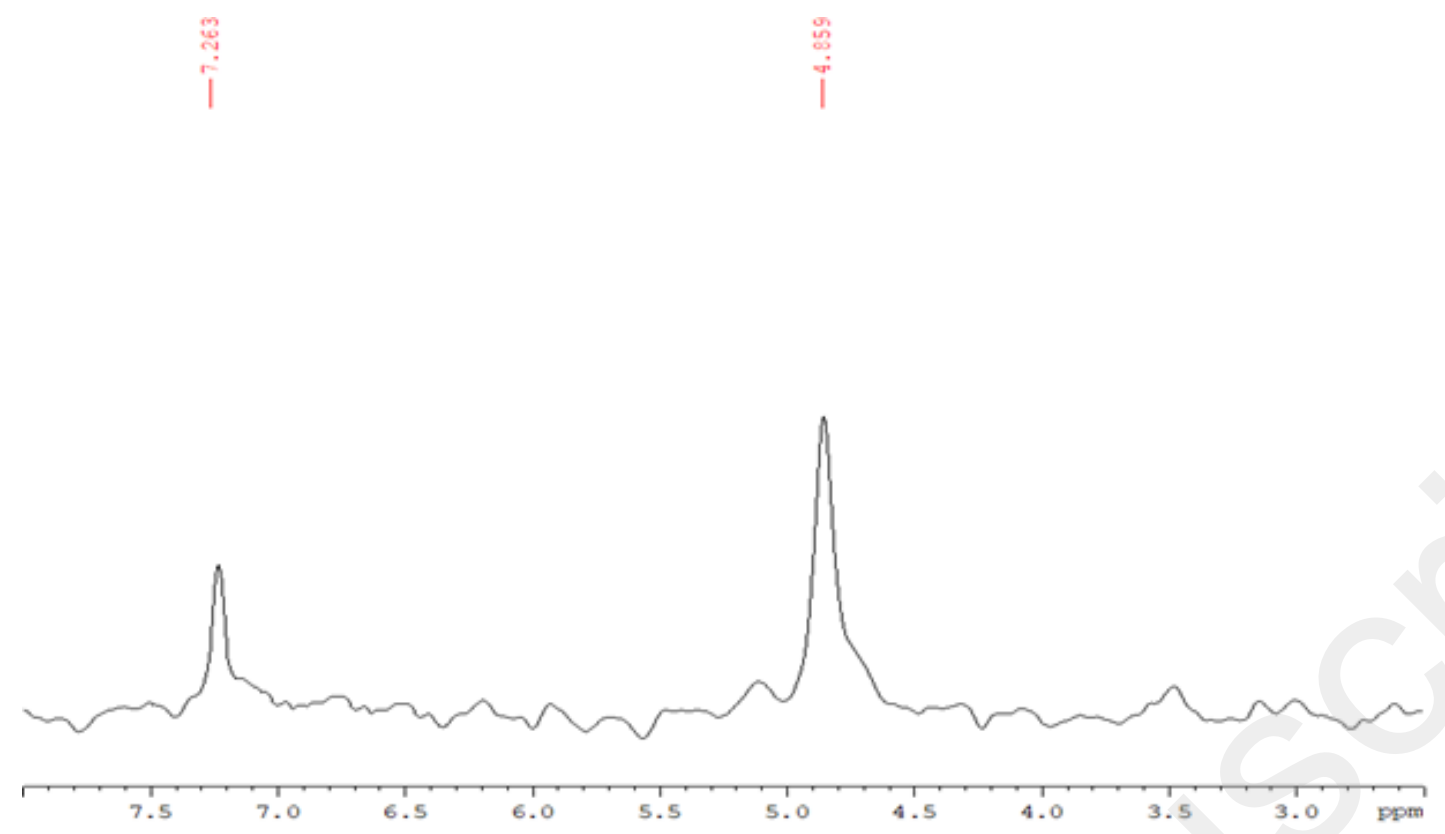

Figure S8. ${ }^{2} \mathrm{H}$ NMR spectrum of $\mathbf{2}_{\mathbf{D}}$ in $\mathrm{CHCl}_{3}$. 


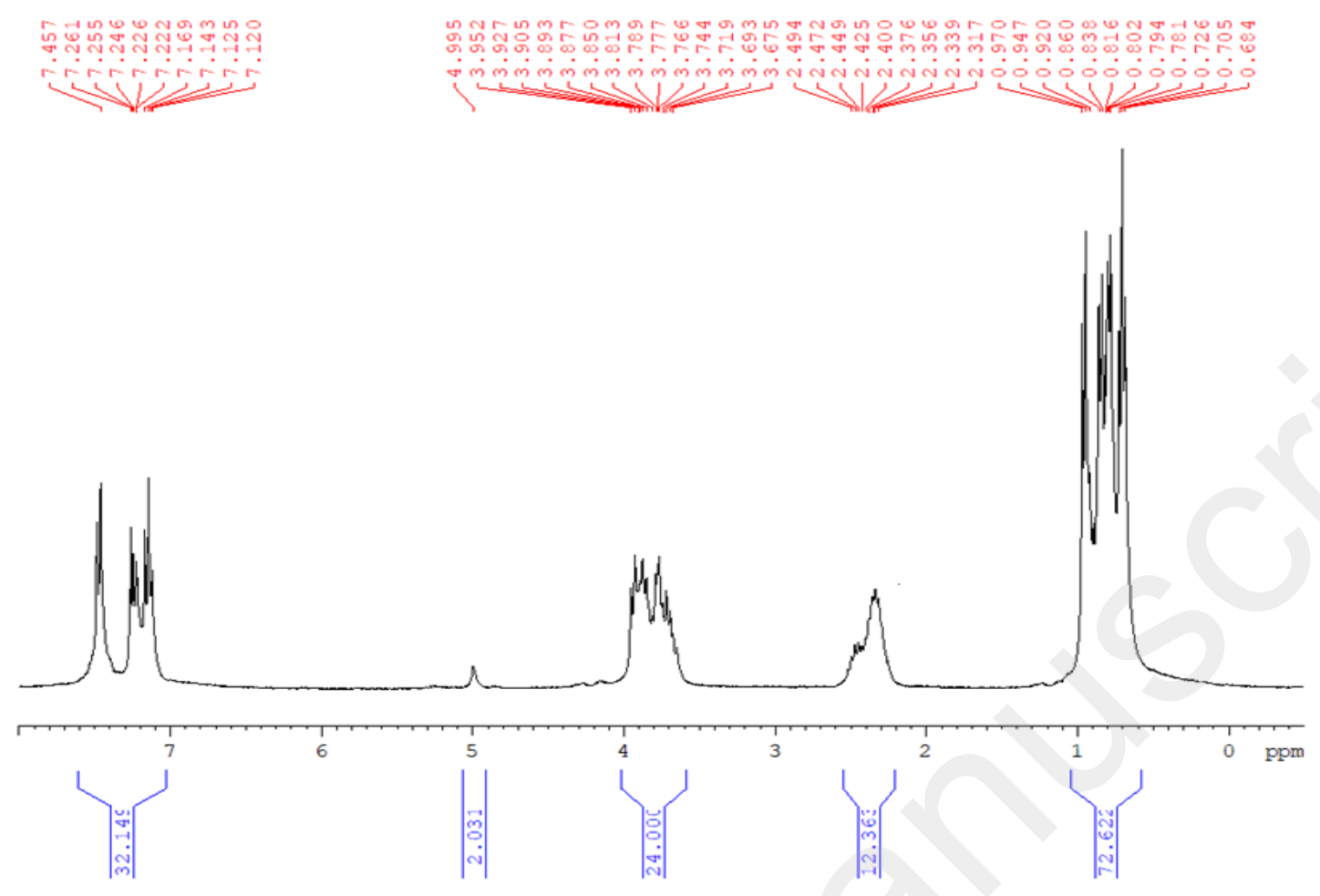

Figure S9. ${ }^{1} \mathrm{H}$ NMR spectrum of $\mathbf{3}_{\mathbf{H}}$ in $\mathrm{CDCl}_{3}$. 


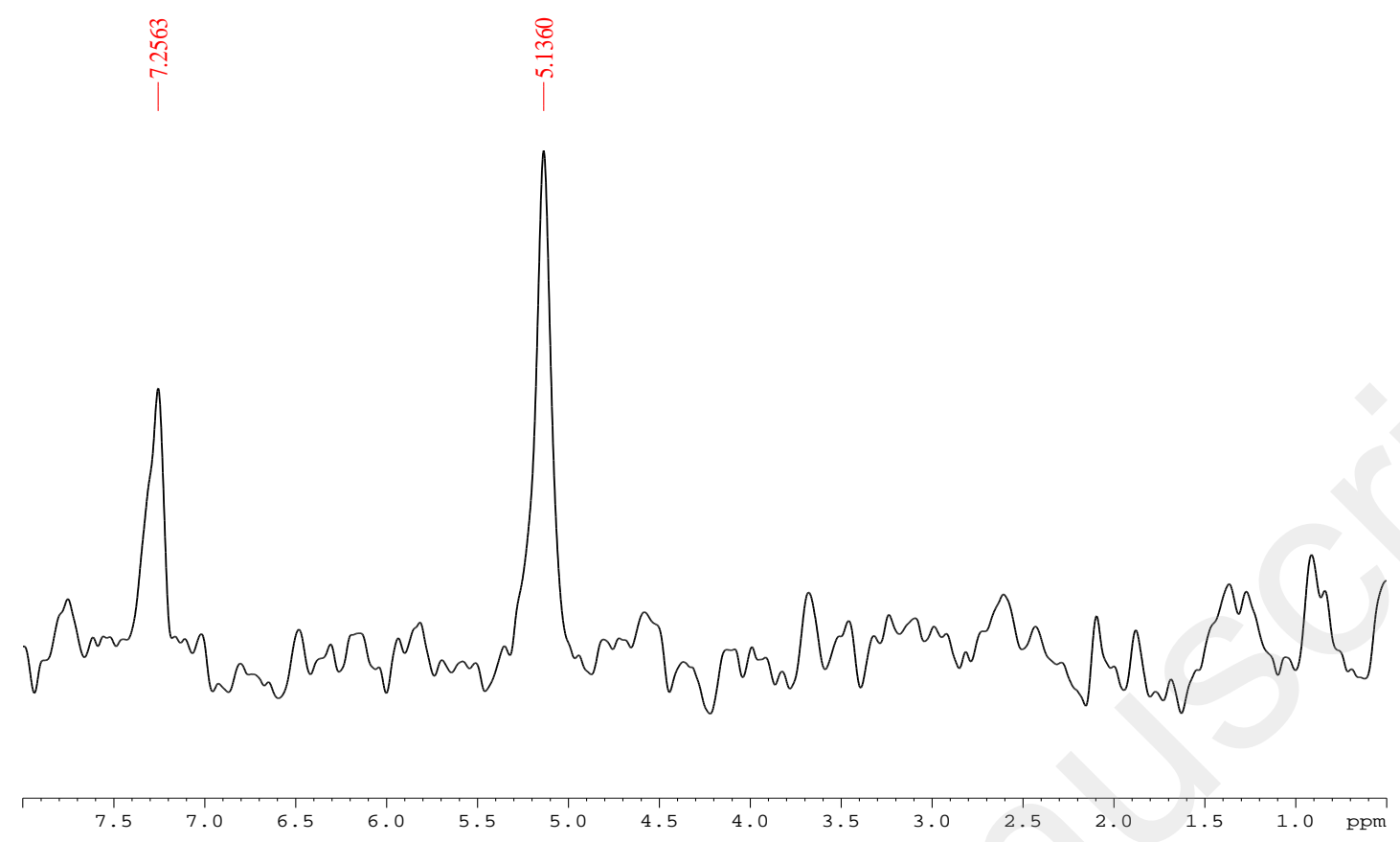

Figure S10. ${ }^{2} \mathrm{H}$ NMR spectrum of $\mathbf{3}_{\mathbf{D}}$ in $\mathrm{CHCl}_{3}$. 


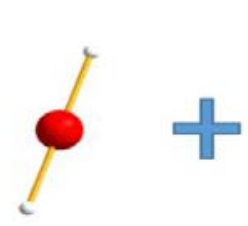

a.

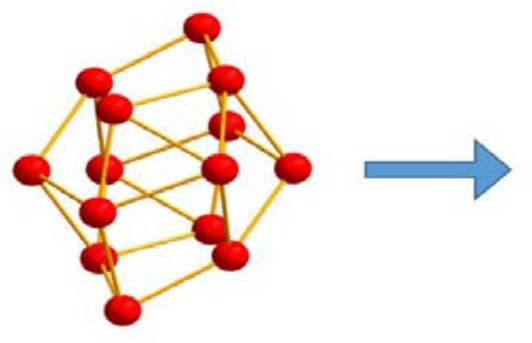

b.

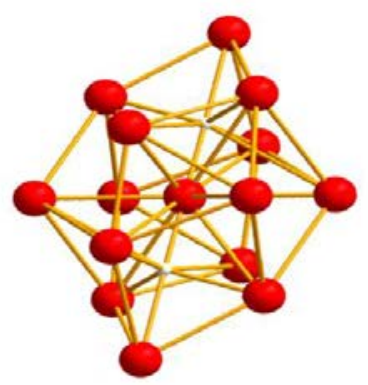

C.

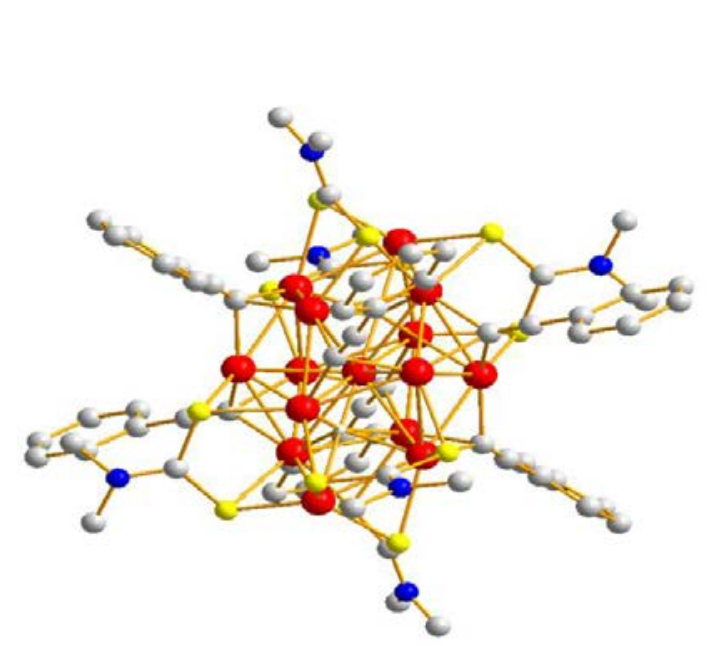

e.
늠

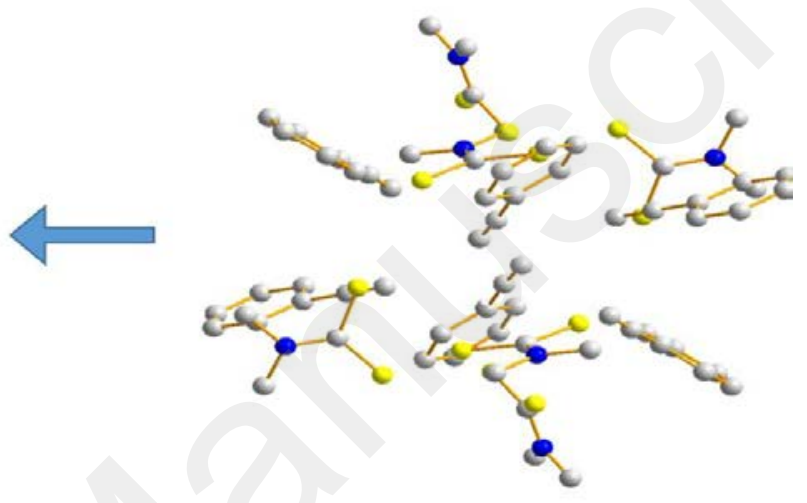

d.

Figure S11. Shell by shell construction of cationic cluster $\left.\left[\mathrm{Cu}_{15}(\mathrm{H})_{2}\left\{\mathrm{~S}_{2} \mathrm{CNR}_{2}\right\}_{6}\left(\mathrm{C}_{2} \mathrm{Ph}\right)_{6}\right]^{+}: \mathrm{a}\right)$. Linear copper dihydride unit; b). The bicapped icosahederal $\mathrm{Cu}_{14}$ shell; c). $\mathrm{Cu}_{15} \mathrm{H}_{2}$ shell; d.) six dithiocarbamate and six phenylacetylene ligands; e). Structure of $\left[\mathrm{Cu}_{15}(\mathrm{H})_{2}\left\{\mathrm{~S}_{2} \mathrm{CNR}_{2}\right\}_{6}\left(\mathrm{C}_{2} \mathrm{Ph}\right)_{6}\right]^{+}$(Hydrogen atoms of the ligands are deleted for clarity). Color: red, copper; yellow, sulphur; blue, nitrogen; grey, carbon; white, hydrogen. 

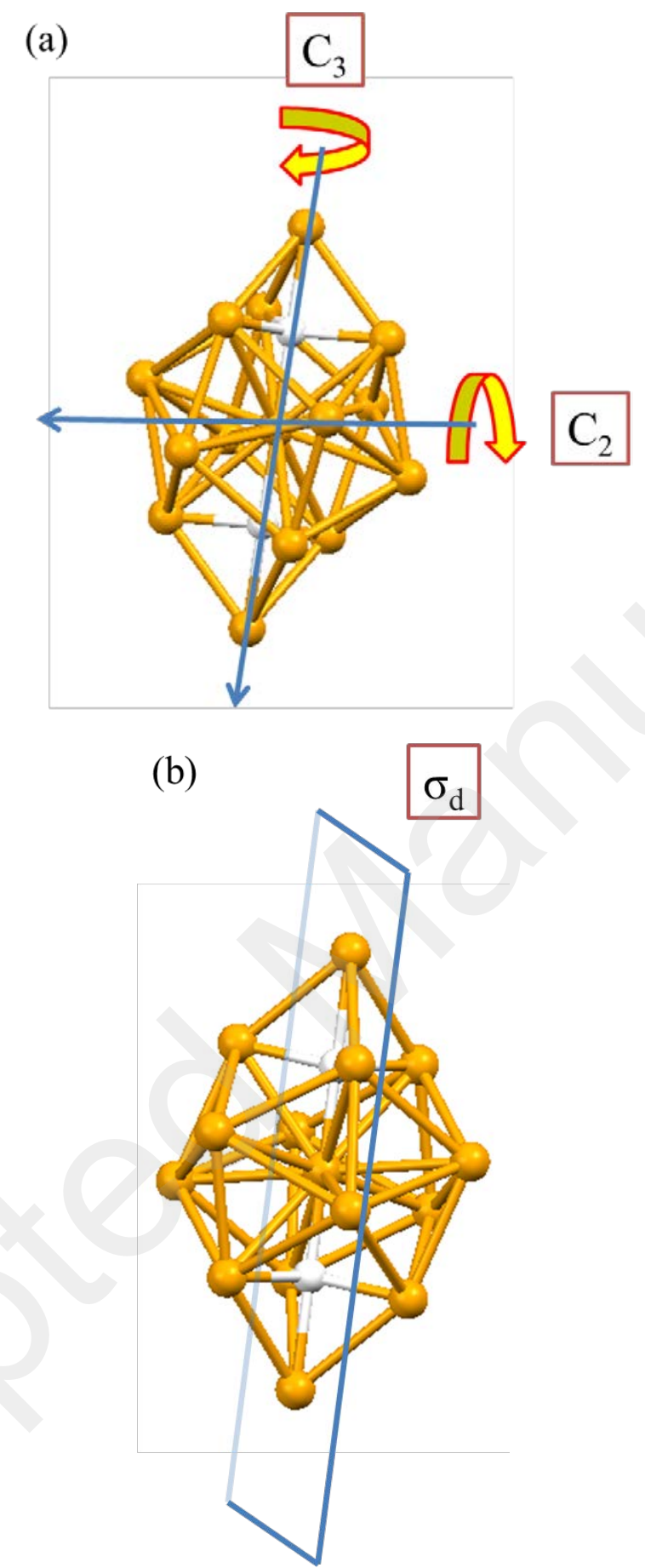

Figure S12. The $\mathrm{D}_{3 d}$ symmetry of a bicapped $\mathrm{Cu}_{15} \mathrm{H}_{2}$ icosahedral along (a) $\mathrm{C}_{3}$ and $\mathrm{C}_{2}$ axis; (b) $\sigma_{\mathrm{d}}$ 


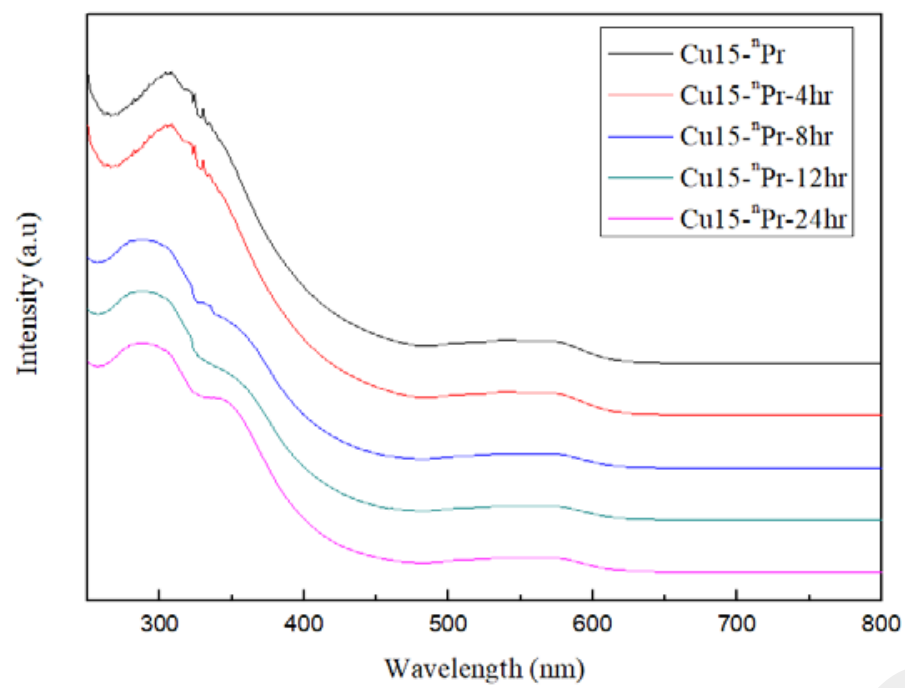

Figure S13. Time dependent UV-visible spectrum for $\left[\mathrm{Cu}_{15}(\mathrm{H})_{2}\left(\mathrm{~S}_{2} \mathrm{CN}\left({ }^{\mathrm{n}} \mathrm{Pr}\right)_{2}\right)_{6}\left(\mathrm{C}_{2} \mathrm{Ph}\right)_{6}\right]\left(\mathrm{PF}_{6}\right)$ change to $\left[\mathrm{Cu}_{13}\left(\mathrm{~S}_{2} \mathrm{CN}\left({ }^{\mathrm{n}} \mathrm{Pr}\right)_{2}\right)_{6}\left(\mathrm{C}_{2} \mathrm{Ph}\right)_{4}\right]\left(\mathrm{PF}_{6}\right)$. 


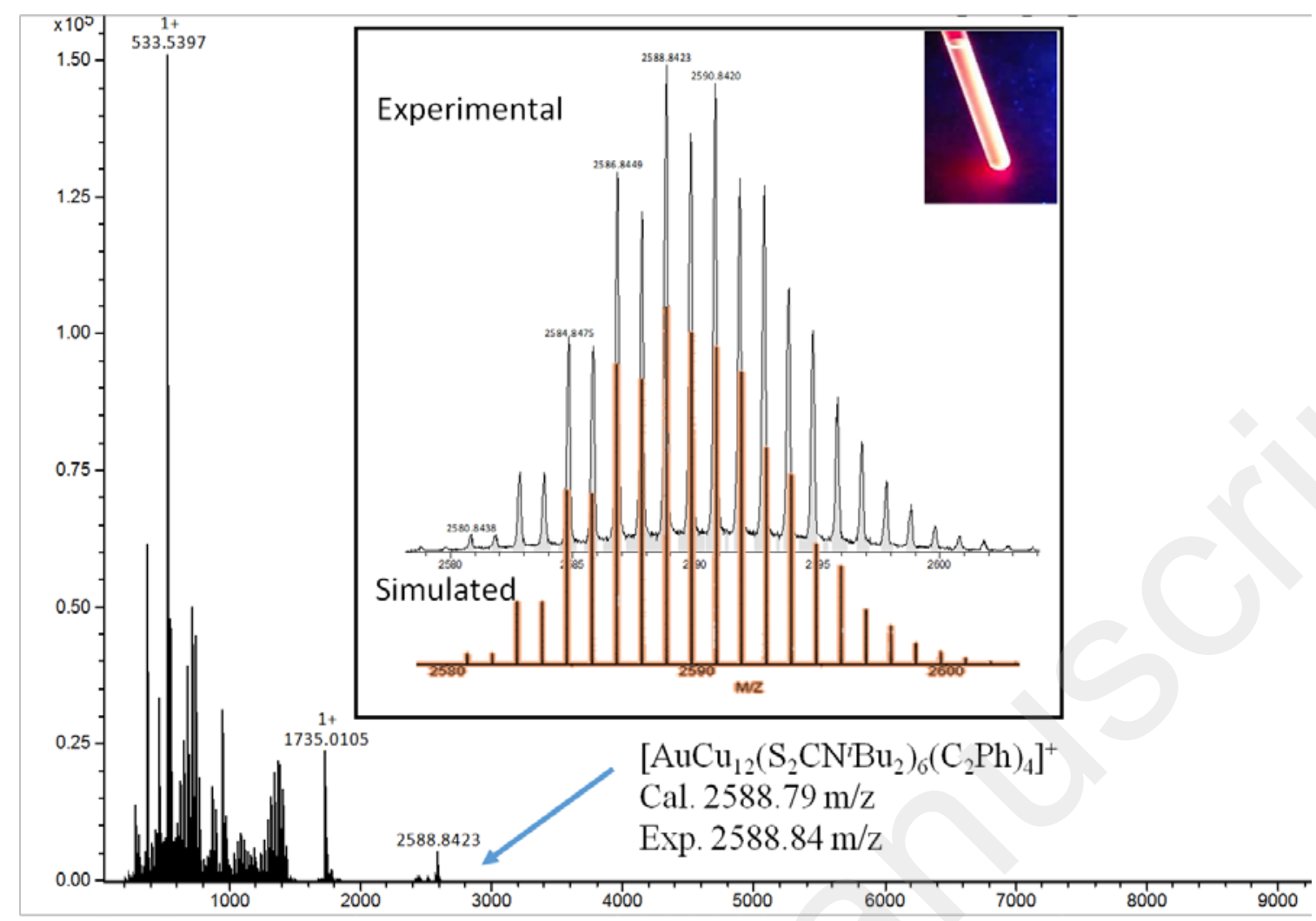

Figure S14. Positive ESI mass spectrum of $\left[\mathrm{AuCu}_{12}\left(\mathrm{~S}_{2} \mathrm{CN}^{i} \mathrm{Bu}_{2}\right)_{6}\left(\mathrm{C}_{2} \mathrm{Ph}\right)_{4}\right]^{+}$isolated from the reaction of $\mathbf{3}_{\mathbf{H}}$ with $\mathrm{Au}\left(\mathrm{PPh}_{3}\right) \mathrm{Cl}$. Inset shows the luminescence behavior of the synthesized complex. 


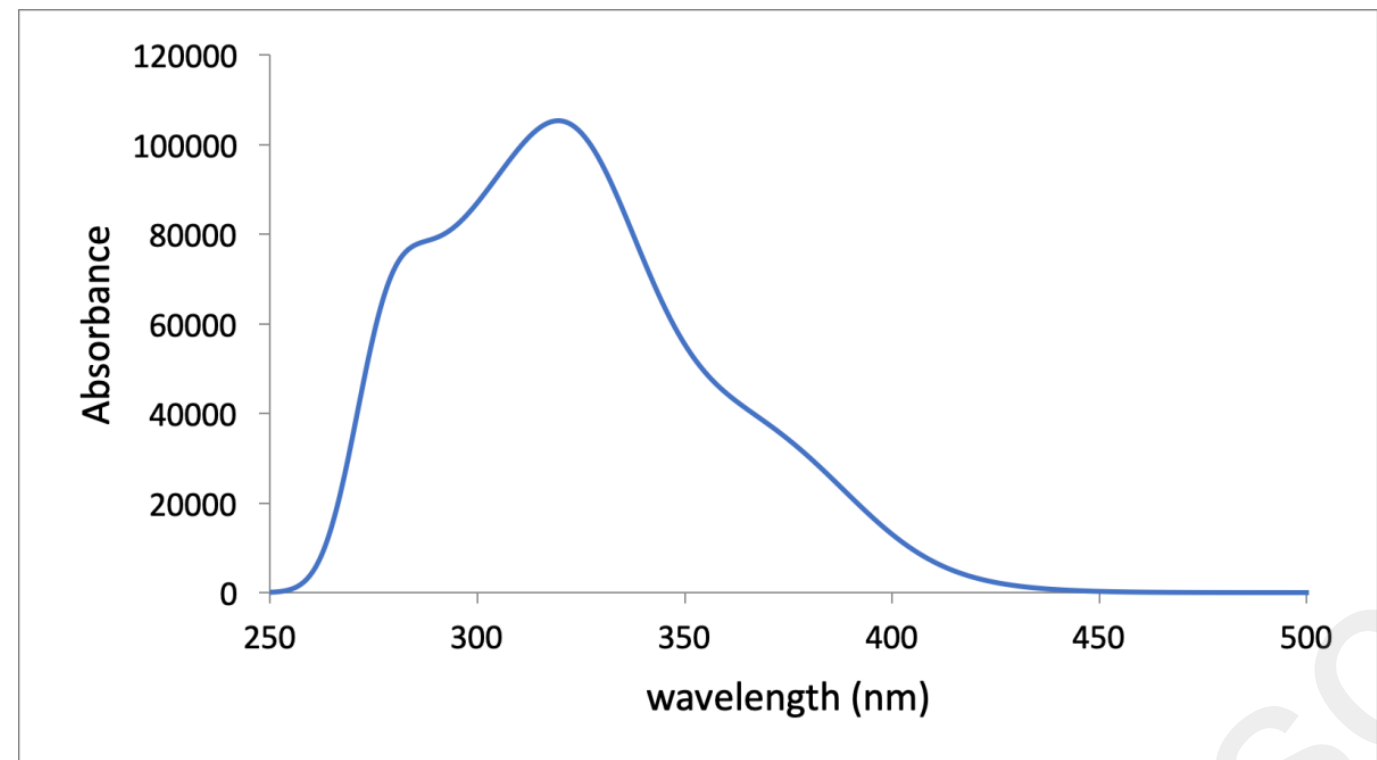

Figure S15. TD-DFT simulated spectrum of 1’. 


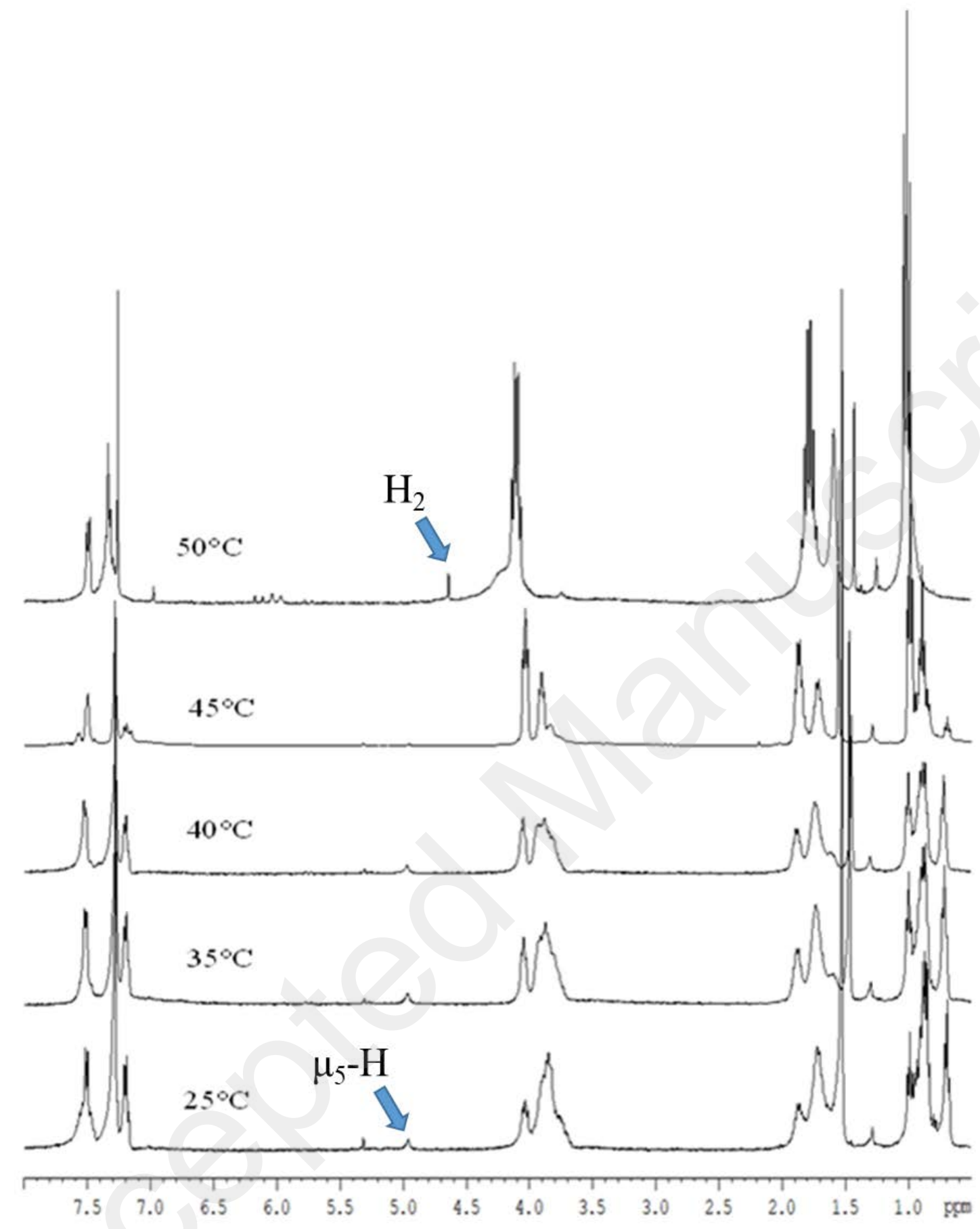

Figure S16. The VT-NMR of $\left[\mathrm{Cu}_{15} \mathrm{H}_{2}\left\{\mathrm{~S}_{2} \mathrm{CN}^{n} \mathrm{Pr}\right\}_{6}\left(\mathrm{C}_{2} \mathrm{Ph}\right)_{6}\right]\left(\mathrm{PF}_{6}\right)$ in $\mathrm{CDCl}_{3}$. 


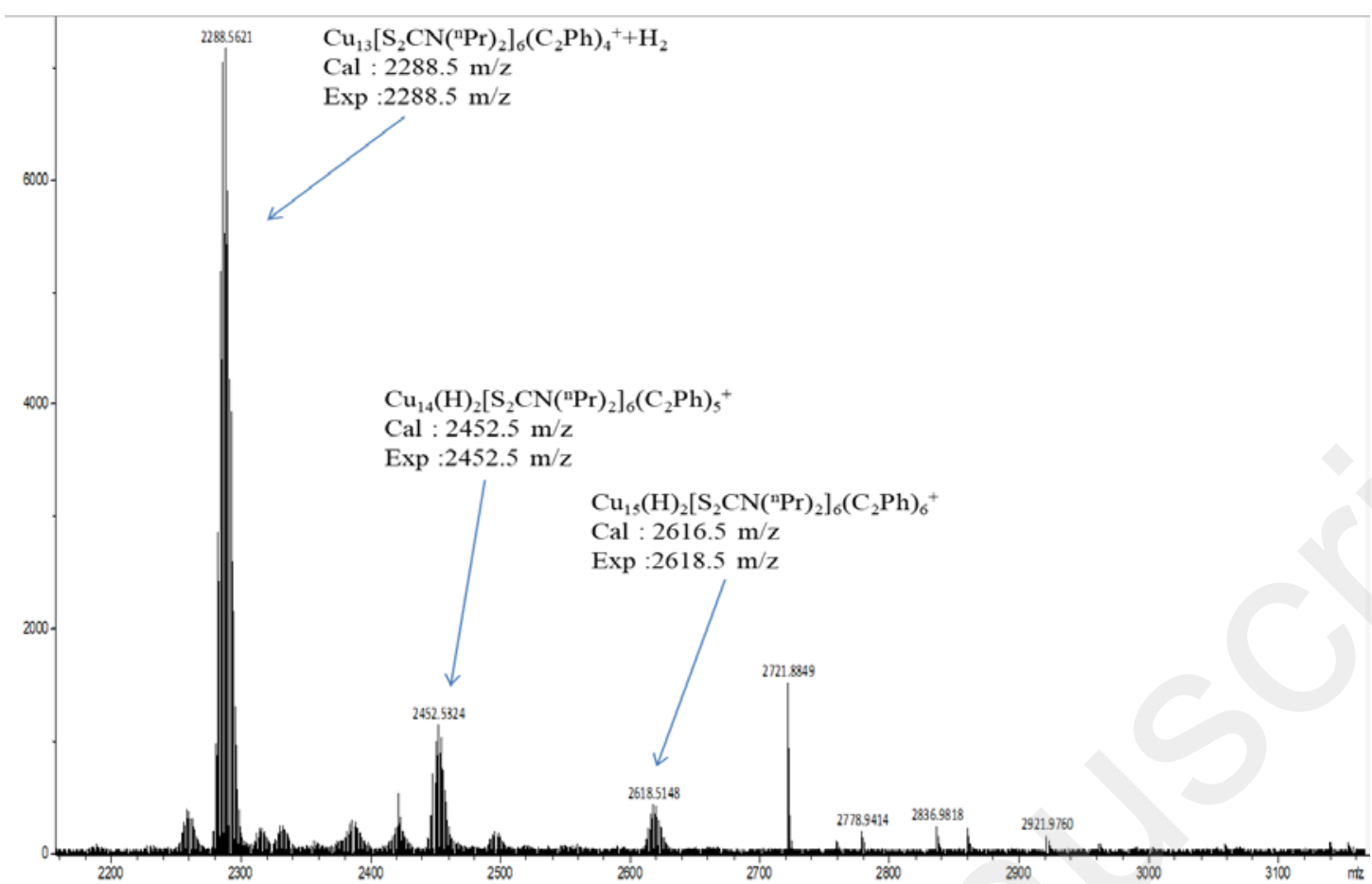

Figure S17. The ESI-MS spectrum showing the decomposition of $\left[\mathrm{Cu}_{15}(\mathrm{H})_{2}\left\{\mathrm{~S}_{2} \mathrm{CNR}_{2}\right\}_{6}\left(\mathrm{C}_{2} \mathrm{Ph}\right)_{6}\right]^{+}$to $\left[\mathrm{Cu}_{13}\left\{\mathrm{~S}_{2} \mathrm{CNR}_{2}\right\}_{6}\left(\mathrm{C}_{2} \mathrm{Ph}_{4}\right)\right]^{+}$cluster. 


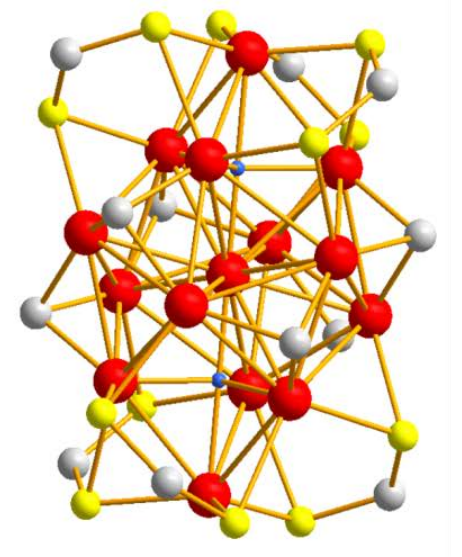

\section{$\mathrm{CH}_{2} \mathrm{Cl}_{2}$ or $\mathrm{CHCl}_{3}$}

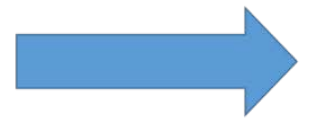

$\Delta$
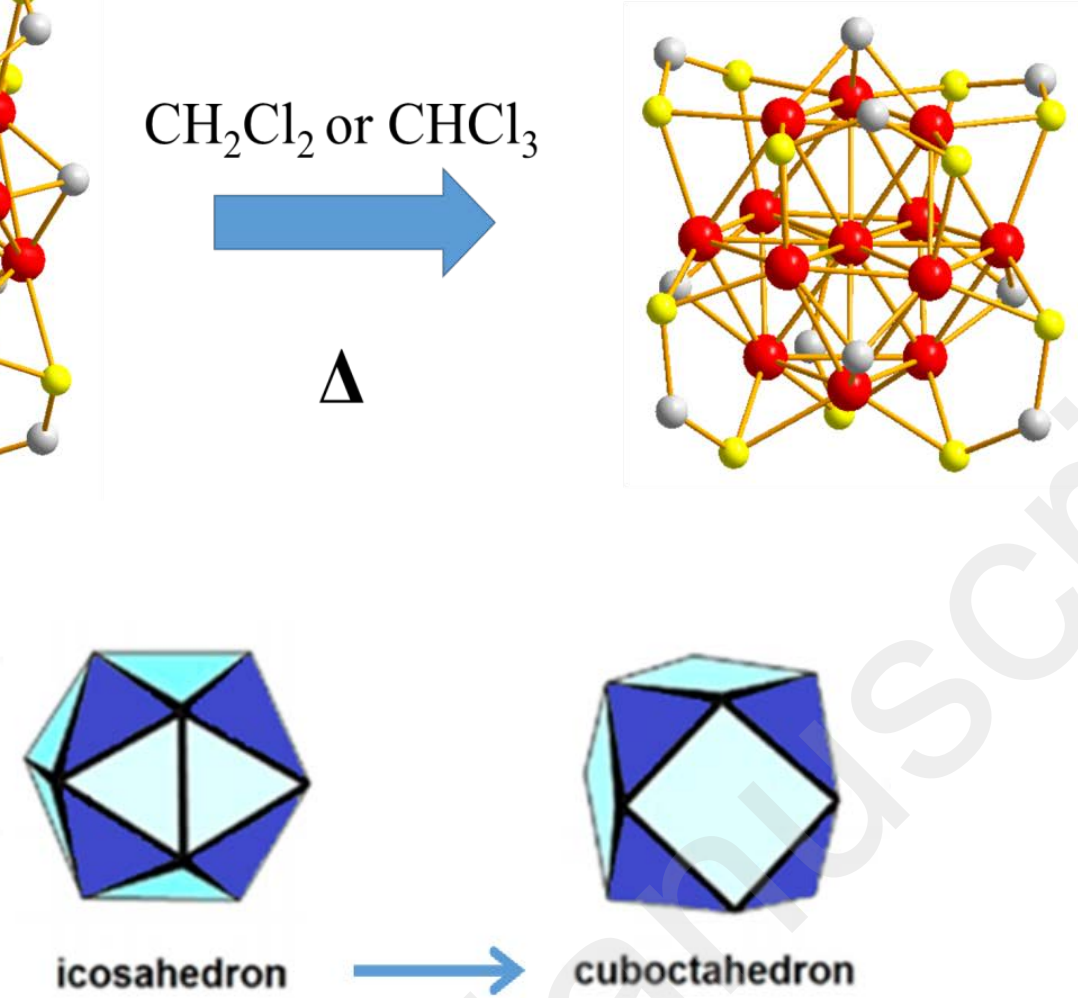

cuboctahedron

$$
\mathrm{Cu}^{+1}+2 \mathrm{H}^{-}
$$

$$
\mathrm{Cu}^{-1}+\mathrm{H}_{2}
$$

Figure S18. Decompositions of $\left[\mathrm{Cu}_{15}(\mathrm{H})_{2}\left\{\mathrm{~S}_{2} \mathrm{CN}^{n} \mathrm{Pr}_{2}\right\}_{6}\left(\mathrm{C}_{2} \mathrm{Ph}\right)_{6}\right]^{+}$in chlorinated solvent en route to $\left[\mathrm{Cu}_{13} \mathrm{H}_{2}\left\{\mathrm{~S}_{2} \mathrm{CN}^{n} \mathrm{Pr}_{2}\right\}_{6}\left(\mathrm{C}_{2} \mathrm{Ph}\right)_{4}\right]^{+}$followed by an internal redox reaction as well as $\mathrm{Cu}_{13}$ core rearrangement to reach a two-electron species. 


\section{REFERENCES}

[1] J.-H. Liao, R. S. Dhayal, X. Wang, S. Kahlal, J.-Y. Saillard, C. W. Liu, Inorg. Chem., 2014, 53, 11140-11145.

[2] C. N. Tsai, S. Mazumder, X. Z. Zhang, H. B. Schlegel, Y. J. Chen, J. F. Endicott, Inorg. Chem., 2015, 54, 8495-8508.

[3] R. A. Thomas, C. N. Tsai, S. Mazumder, I. C. Lu, R. L. Lord, H. B. Schlegel, Y. J. Chen, J. F. Endicott, J. Phys. Chem. B., 2015, 119, 7393-7406.

[4] SAINT V4.043: Software for CCDC Detector System; Bruker Analystic X-ray Systems, Madison, WI, 1995.

[5] G. M. Sheldrick, SADABS, University of Göttingen, Göttingen, Germany, 1996.

[6] G. M. Sheldrick, A short history of SHELX. Acta Cryst. 2008, A64, 112-122.

[7] SHELXL v6.14 (PC version) Program Library for Structure Solution and Molecular Graphics, Bruker Analytical X-ray Systems, Madison, WI, 1998.

[8] Z. Xue, A. Ramirez-Cuesta, C. Brown, S. Calder, H. Cao, B. Chakoumakos, L. Daemen, A. Huq, A. Kolesnikov, E. Mamontov, A. Podlesnyak, X. Wang, Eur. J. Inorg. Chem. 2019, 1065-1089.

[9] J. Zikovsky, P. F. Peterson, X. P. Wang, M. Frost, C. Hoffmann, J. Appl. Cryst., 2011, 44, 418-423.

[10] A. J. Schultz, M. R. V. Jorgensen, X. Wang, R. L. Mikkelson, D. J. Mikkelson, V. E. Lynch, P. F. Peterson, M. L. Green, C. M. Hoffmann, J. Appl. Cryst., 2014, 47, 915-921.

[11] G. M. Sheldrick, ActaCryst., 2015, C71, 3-8.

[12] Gaussian 16, Revision C.01, Frisch, M. J.; Trucks, G. W.; Schlegel, H. B.; Scuseria, G. E.; Robb, M. A.; Cheeseman, J. R.; Scalmani, G.; Barone, V.; Petersson, 
G. A.; Nakatsuji, H.; Li, X.; Caricato, M.; Marenich, A. V.; Bloino, J.; Janesko, B. G.;

Gomperts, R.; Mennucci, B.; Hratchian, H. P.; Ortiz, J. V.; Izmaylov, A. F.;

Sonnenberg, J. L.; Williams-Young, D.; Ding, F.; Lipparini, F.; Egidi, F.; Goings, J.;

Peng, B.; Petrone, A.; Henderson, T.; Ranasinghe, D.; Zakrzewski, V. G.; Gao, J.;

Rega, N.; Zheng, G.; Liang, W.; Hada, M.; Ehara, M.; Toyota, K.; Fukuda, R.;

Hasegawa, J.; Ishida, M.; Nakajima, T.; Honda, Y.; Kitao, O.; Nakai, H.; Vreven, T.;

Throssell, K.; Montgomery, J. A., Jr.; Peralta, J. E.; Ogliaro, F.; Bearpark, M. J.; Heyd,

J. J.; Brothers, E. N.; Kudin, K. N.; Staroverov, V. N.; Keith, T. A.; Kobayashi, R.;

Normand, J.; Raghavachari, K.; Rendell, A. P.; Burant, J. C.; Iyengar, S. S.; Tomasi,

J.; Cossi, M.; Millam, J. M.; Klene, M.; Adamo, C.; Cammi, R.; Ochterski, J. W.;

Martin, R. L.; Morokuma, K.; Farkas, O.; Foresman, J. B.; Fox, D. J. Gaussian, Inc., Wallingford CT, 2016.

[13] C. Adamo, A.; Barone, V. Toward reliable density functional methods without adjustable parameters: The PBE0 model. J. Chem. Phys. 1999, 110, 6158-69.

[14] F. Weigend, F.; Ahlrichs, R. Balanced basis sets of split valence, triple zeta valence and quadruple zeta valence quality for $\mathrm{H}$ to $\mathrm{Rn}$ : Design and assessment of accuracy. Phys. Chem. Chem. Phys. 2005, 7, 3297-305.

[15] Glendening, E.D.; Badenhoop, J. K.; Reed, A. E.; Carpenter, J. E.; Bohmann, J. A.; Morales, C. M.; Landis, C. R.; Weinhold, F. NBO 6.0; Theoretical Chemistry Institute, University of Wisconsin, Madison, WI, 2013, http://nbo6.chem.wisc.edu. [16] Ullrich, C. Time-Dependent Density-Functional Theory, Concepts and Applications, Oxford University Press, NewYork, 2012.

[17] Gorelsky, S. I. SWizard program, revision 4.5, http://www.sg-chem.net.

[18] Gorelsky, S. I. AOMix program, http://www.sg-chem.net. 
[19] K. K. Chakrahari, R. P. B. Silalahi, T.-H. Chiu, X. Wang, N. Azrou, S. Kahlal, Y.-C. Liu, M.-H. Chiang, J.-Y. Saillard, C. W. Liu, Angew. Chem. Int. Ed., 2019, 58, $4943-4947$. 